\title{
Multi-Channel Atomic Scattering and Confinement-Induced Resonances in Waveguides
}

\author{
Shahpoor Saeidian \\ Physikalisches Institut, Universität Heidelberg, \\ Philosophenweg 12, 69120 Heidelberg, Germany \\ Vladimir S.Melezhik \\ Bogoliubov Laboratory of Theoretical Physics, \\ Joint Institute for Nuclear Research, Dubna, \\ Moscow Region 141980, Russian Federation \\ Peter Schmelcher \\ Physikalisches Institut, Universität Heidelberg, \\ Philosophenweg 12, 69120 Heidelberg, Germany and \\ Theoretische Chemie, Institut für Physikalische Chemie, \\ Universität Heidelberg, INF 229, 69120 Heidelberg, Germany
}

(Dated: October 22, 2018) 


\begin{abstract}
We develop a grid method for multi-channel scattering of atoms in a waveguide with harmonic confinement. This approach is employed to extensively analyze the transverse excitations and deexcitations as well as resonant scattering processes. Collisions of identical bosonic and fermionic as well as distinguishable atoms in harmonic traps with a single frequency $\omega$ permitting the centerof-mass (c.m.) separation are explored in depth. In the zero-energy limit and single mode regime we reproduce the well-known confinement-induced resonances (CIRs) for bosonic, fermionic and heteronuclear collisions. In case of the multi-mode regime up to four open transverse channels are considered. Previously obtained analytical results are extended significantly here. Series of Feshbach resonances in the transmission behaviour are identified and analyzed. The behaviour of the transmission with varying energy and scattering lengths is discussed in detail. The dual CIR leading to a complete quantum suppression of atomic scattering is revealed in multi-channel scattering processes. Possible applications include, e.g., cold and ultracold atom-atom collisions in atomic waveguides and electron-impurity scattering in quantum wires.
\end{abstract}

PACS numbers: $34.10 .+\mathrm{x}, 03.75 . \mathrm{Be}, 34.50 .-\mathrm{s}$

\footnotetext{
*s:saeid@physi.uni-heidelberg.de

melezhik@thsun1.jinr.ru

Peter.Schmelcher@pci.uni-heidelberg.de
} 


\section{INTRODUCTION}

During the last years, the field of ultracold few-body confined systems has progressed remarkably. By employing optical dipole traps [1] and atom chips [2, 3, 4] it is possible to fabricate mesoscopic structures in which the atoms are freezed to occupy a single or a few lowest quantum states of a confining potential such that in one or more dimensions the characteristic length possesses the order of the atomic deBroglie wavelength. These configurations can be well described by effective one-dimensional systems. Well-known examples are quantum wires and atom waveguides or quasi two-dimensional systems such as 2D electronic gases. The quantum dynamics of such systems is strongly influenced by the geometry of the confinement.

To control the coherent propagation of particle beams through $1 \mathrm{D}$ or $2 \mathrm{D}$ traps it is crucial to very well understand the impact of the confinement on the collisional properties (see refs. [5, 6, 17, 8, 9, 10, 11, 12, 13, 14, 15, 16, 17, 18, 19, 20, 21, 22, 23, 24, 25, 26, 27, 28, 29] and refs.therein). Free-space scattering theory is no longer valid in such systems and a new theory is needed. This stimulated the development of quantum scattering theory in low dimensions. For a sufficiently dilute gas under a strong transverse confinement, one may expect both the chemical potential and the thermal energy $k_{B} T$ being less than the transverse level spacing. With this assumption, the dynamics of ultracold atoms in low dimensional structures e.g. tight wave guides has been studied using the simplification that the atoms occupy only the ground state of the transverse confining potential. Nevertheless, the virtual transverse excitations in the course of the collision process can play a crucial role in the scattering leading to the so-called confinement-induced resonances (CIRs), predicted by Olshanii [13, 14]. S-wave zero-energy scattering of bosons is mapped to an effective longitudinal zerorange pseudopotential $g_{1 D} \delta(z)$ approximating the 1D atom-atom interaction in a transverse harmonic confinement. It was shown[13, 14] that CIRs appear if the binding energy of the pseudopotential, approximating the two-atom molecular state in the presence of the confinement, coincides with the energy spacing between the levels of the transverse harmonic potential. In the vicinity of the CIR the coupling constant $g_{1 D}$ can be tuned from $-\infty$ to $+\infty$ by varying the strength of the confining potential over a small range. This can result in a total atom-atom reflection, thereby creating a gas of impenetrable bosons. CIRs have also been studied for the three-body [15, 16] and the four-body [17] scattering under 
confining potential, as well as for a pure p-wave scattering of fermions [18]. Experimental evidence for the CIRs for bosons [19, 20] and fermions [21] has recently been reported. A general analytical treatment of low-energy scattering under action of a general cylindrical confinement involving all partial waves and their coupling, was first provided in ref.[22] for a spherically symmetric short-range potential. The effect of the c.m. motion on the s-wave collision of two distinguishable atoms (i.e. a two-species mixture) in a harmonic confinement as well as for two identical atoms in a non-parabolic confining potential has been investigated in ref.[23] in the zero-energy limit neglecting the s and p wave mixing. A detailed study including the effect of the c.m. nonseparability and taking into account the $\mathrm{s}$ and $\mathrm{p}$ wave mixing for harmonic confinement was performed in the single-mode regime in ref. [26]. Recently a so-called dual-CIR was discovered [24, 25, 26], which is characterized by a complete transmission (suppression of quantum scattering) in the waveguide due to destructive interference of $\mathrm{s}$ and $\mathrm{p}$ waves although the corresponding collisions in free space involve strong interactions.

The problem of atomic pair collisions under the action of a harmonic trap in the multimode regime when the energy of the atoms exceeds the level spacing of the transverse trapping potential is much more intricate than the single-mode regime due to several open transverse channels. It demands the development of a multi-channel scattering theory accounting for the possible transitions between the levels of the confining potential. Using as a starting point the formalism for scattering in restricted geometries suggested in refs. [27, 28], the multi-channel scattering problem for bosons in a harmonic confining potential has been analyzed analytically by Olshanii el al. [29] in the s-wave pseudopotential approximation and the zero-energy limit. Without detailization of the interatomic interaction but using only two input parameters - the s-wave two-body scattering length in free space and the trap frequency - they have derived an approximate formula for the scattering amplitude describing two boson collisions confined by transverse harmonic trap in the multi-mode regime.

In the present work we develop a general grid method for multi-channel scattering of identical as well as distinguishable atoms confined by a transverse harmonic trap. The method applies to arbitrary atomic interactions, permitting a rich spectral structure where several different partial waves are participating in the scattering process or even the case of an anisotropy of the interaction. The only limitation is that we consider harmonic traps with 
a single frequency for every atom causing a separation of the c.m. and relative motion. With our approach we analyze transverse excitations/deexcitations in the course of the collisional process (distinguishable or identical atoms) including all important partial waves and their couplings due to the broken spherical symmetry. Special attention is paid to the analysis of the CIRs in the multi-mode regimes for non-zero collision energies, i.e., to suggest a nontrivial extension of the CIRs theory developed so far only for the single-mode regime and zero-energy limit.

In detail we proceed as follows. Section II contains the derivation of the Hamiltonian, definitions of the interatomic interaction and the scattering asymptotics in the confined geometry, and a discussion of the scattering parameters and the transition probabilities characterizing the two-body collisions in the trap. Our computational method is outlined in Sec. III, important technical details are given in the Appendix. In section IV our results are presented and analyzed. A summary and conclusions are given in Sec. V.

\section{HAMILTONIAN AND TWO-BODY SCATTERING PROBLEM IN A WAVEG- UIDE}

Let us consider collisions of two atoms under the action of the transverse harmonic confinement. We address both cases, distinguishable and indistinguishable atoms under the action of the same confining potential, i.e. the trap is characterized by a single frequency $\omega$ for every atom. The corresponding Hamiltonian is given by

$$
H=-\frac{\hbar^{2}}{2 m_{1}} \nabla_{1}^{2}-\frac{\hbar^{2}}{2 m_{2}} \nabla_{2}^{2}+\frac{1}{2} m_{1} \omega^{2} \rho_{1}^{2}+\frac{1}{2} m_{2} \omega^{2} \rho_{2}^{2}+V\left(\mathbf{r}_{1}-\mathbf{r}_{2}\right)
$$

where $m_{i}$ is the mass of the $i-t h$ atom, $V\left(\mathbf{r}_{1}-\mathbf{r}_{2}\right)$ is the two-body potential describing the interaction between two colliding atoms in free space and $\mathbf{r}_{i}=\left(\rho_{i}, z_{i}\right)=\left(r_{i}, \theta_{i}, \phi_{i}\right)$ are the coordinates of the $i-t h$ atom. The Hamiltonian is separable with respect to the relative $\mathbf{r}=\mathbf{r}_{1}-\mathbf{r}_{2}$ and c.m. $\mathbf{R}=\left(m_{1} \mathbf{r}_{1}+m_{2} \mathbf{r}_{2}\right) /\left(m_{1}+m_{2}\right)$ variables

$$
H=H_{C M}+H_{R e l},
$$

where

$$
H_{C M}=-\frac{\hbar^{2}}{2 M} \nabla_{R}^{2}+\frac{1}{2} M \omega^{2} \rho_{R}^{2},
$$


and

$$
H_{R e l}=-\frac{\hbar^{2}}{2 \mu} \nabla_{r}^{2}+\frac{1}{2} \mu \omega^{2} \rho^{2}+V(r) .
$$

Here $M=m_{1}+m_{2}$ and $\mu=m_{1} m_{2} /\left(m_{1}+m_{2}\right)$ are the total and reduced masses respectively. The problem, thus, reduces to scattering of a single effective particle with the reduced mass $\mu$ and collision energy $\epsilon>\hbar \omega$, off a scatterer $V(r)$ at the origin, under transverse harmonic confinement with frequency $\omega$

$$
\left[-\frac{\hbar^{2}}{2 \mu} \nabla_{r}^{2}+\frac{1}{2} \mu \omega^{2} \rho^{2}+V(r)\right] \psi(\mathbf{r})=\epsilon \psi(\mathbf{r}) .
$$

Here the energy of the relative two-body motion $\epsilon=\epsilon_{\perp}+\epsilon_{\|}$is a sum of the transverse $\epsilon_{\perp}$ and longitudinal collision $\epsilon_{\|}$energies. Due to our definition of the confining potential, the transverse excitation energies $\epsilon_{\perp}$ can take the possible values $\epsilon_{\perp}=\epsilon-\epsilon_{\|}=\hbar \omega(2 n+|m|+1)>$ 0 of the discrete spectrum of the $2 \mathrm{D}$ oscillator $\frac{1}{2} \mu \omega^{2} \rho^{2}$.

For the two-body interaction we choose a screened Coulomb potential

$$
V(r)=V_{0} \frac{r_{0}}{r} e^{-r / r_{0}},
$$

already employed in ref.[24, 25, 26] for analyzing the ultracold scattering in cylindrical waveguides in the single-mode regime as $\epsilon_{\perp}=\epsilon-\epsilon_{\|}$. The chosen potential (6) depends on two parameters - the depth $V_{0}<0$ and the screening length $r_{0}>0$. Following the computational scheme already developed in ref.[26], we implement different spectral structures of the atomic interaction by varying the single parameter $V_{0}$ for a fixed length scale $r_{0}$. Obvious advantages of the screened Coulomb potential (6) compared to the s-wave pseudopotential used in ref. [29] that is devoted to the multi-channel scattering of bosons are the following. First, by varying $V_{0}$ one can vary the number of bound states of s-wave character in the interaction potential, and second, one can create new bound and resonant states of higher partial wave character. As a consequence we can consider not only bosonic but also fermionic as well as mixed collisions in a trap and including the case of higher energies. This will, as we shall see below, permit us to investigate new regimes and effects of multi-channel confined scattering.

Performing the scale transformation

$$
r \rightarrow \frac{r}{a_{0}} \quad, \quad \epsilon \rightarrow \frac{\epsilon}{\epsilon_{0}} \quad, \quad V_{0} \rightarrow \frac{V_{0}}{\epsilon_{0}} \quad \text { and } \quad \omega \rightarrow \frac{\omega}{\omega_{0}}
$$

with the units $a_{0}=\hbar^{2} / \mu V_{0} r_{0}, \epsilon_{0}=\hbar^{2} / \mu a_{0}^{2}$, and $\omega_{0}=\epsilon_{0} / \hbar$, it is convenient to rewrite the equation (5) in the rescaled form

$$
\left[-\frac{1}{2} \nabla_{r}^{2}+\frac{1}{2} \omega^{2} \rho^{2}+V(r)\right] \psi(\mathbf{r})=\epsilon \psi(\mathbf{r}),
$$


where $V$ is now the correspondingly scaled potential and we fix $\mu=1$ and $r_{0}=1$ in the subsequent consideration. In the asymptotic region, $|z| \rightarrow \infty$, where the transverse trapping potential dominates the interaction potential, the axial and transverse motions decouple and the asymptotic wavefunction can be written as a product of the longitudinal and transverse $\phi_{n, m}(\rho, \varphi)$ components with

$$
\phi_{n, m}(\rho, \varphi)=\left[\frac{\pi a_{\perp}^{2}(n+|m|) !}{n !}\right]^{-1 / 2}\left(\frac{\rho}{a_{\perp}}\right)^{|m|} e^{-\rho^{2} /\left(2 a_{\perp}^{2}\right)} L_{n}^{|m|}\left(\rho^{2} / a_{\perp}^{2}\right) e^{i m \varphi}
$$

being the eigenfunctions of the transverse trapping Hamiltonian

$$
H_{\perp}=-\frac{1}{2}\left(\frac{\partial^{2}}{\partial \rho^{2}}+\frac{1}{\rho} \frac{\partial}{\partial \rho}+\frac{1}{\rho^{2}} \frac{\partial^{2}}{\partial \varphi^{2}}\right)+\frac{1}{2} \omega^{2} \rho^{2},
$$

with the corresponding eigenvalues $\epsilon_{\perp}=\omega(2 n+|m|+1)$ and the angular momentum projection $m$ onto the z-axis. Here $L_{n}^{m}(x)$ are the generalized Laguerre polynomials and $a_{\perp}=1 / \sqrt{\omega}$, is the transverse oscillator length. The radial and azimuthal quantum numbers $n$ and $m$ of the $2 \mathrm{D}$ harmonic oscillator independently take the values $n=0,1,2, \ldots \infty$ and $m=0, \pm 1, \pm 2, \ldots \pm \infty$. Due to the axial symmetry of the transverse confinement and the spherical symmetry of the interatomic interaction (6) the angular momentum component along the $z$-axis is conserved. Therefore, the problem (8) is reducible to a $2 \mathrm{D}$ one by separating the $\varphi$-variable and can be solved for every $m$ independently. The quantum number $n$ is a good one only in the asymptotic region $|z| \rightarrow \infty$ and used for the definition of the initial (incident) asymptotic state i.e. channel

$$
\psi_{n, m}^{i n}(\mathbf{r})=e^{i k_{n} z} \phi_{n, m}(\rho, \varphi)
$$

of two infinitely separated atoms confined in a transverse state $<\rho \varphi \mid n m>$. In addition to the quantum numbers $n$ and $m$ the asymptotic scattering state is defined also by the momentum $k_{n}$ of the channel

$$
k_{n}=\frac{2}{a_{\perp}} \sqrt{\varepsilon-n-\frac{|m|}{2}},
$$

which we express through the dimensionless energy $\varepsilon=\epsilon /(2 \omega)-1 / 2$. It is clear that the integer part of the dimensionless energy $\varepsilon$ coincides with the number $n_{e}$ of open excited transverse channels. For $n \leq n_{e}$ we have $\left(\varepsilon-n-\frac{|m|}{2}\right)>0$. The spherically symmetric interatomic interaction (6) mixes different transversal channels and leads to transitions $n \rightarrow$ 
$n^{\prime}$ between the open channels $n, n^{\prime} \leq n_{e}$, i.e. to transverse excitation/deexcitation processes during the collisions.

Assuming the system to be initially in the channel $n$, the asymptotic wavefunction takes at $|z| \rightarrow+\infty$ the form [29]

$$
\psi_{n, m}(\mathbf{r})=e^{i k_{n} z} \phi_{n, m}(\rho, \varphi)+\sum_{n^{\prime}=0}^{n_{e}}\left[f_{n n^{\prime}}^{e}+\operatorname{sgn}(z) f_{n n^{\prime}}^{o}\right] e^{i k_{n^{\prime}}|z|} \phi_{n^{\prime}, m}(\rho, \varphi),
$$

where $f_{n n^{\prime}}^{e}$ and $f_{n n^{\prime}}^{o}$ are the matrix elements of the inelastic scattering amplitudes for the even and odd partial waves, respectively, which describe transitions between the channels $n$ and $n^{\prime}$. For a bosonic (fermionic) collision just the symmetric (antisymmetric) part of (13) should be considered.

It is clear that the scattering amplitude depends also on the index $m$ which, however, remains unchanged during the collision due to the axial symmetry of the problem. Hereafter we consider only the case $m=0$ and the index is omitted in the following.

Using the asymptotic wavefunction (13) and the total current conservation one obtains

$$
\sum_{n^{\prime}=0}^{n_{e}}\left(T_{n n^{\prime}}+R_{n n^{\prime}}-\delta_{n n^{\prime}}\right)=0
$$

for the inelastic transmission (reflection) coefficients $T_{n n^{\prime}}\left(R_{n n^{\prime}}\right) . k_{n}\left(k_{n^{\prime}}\right)$ is the initial (final) relative wave vector and $n\left(n^{\prime}\right)$ the transverse excitation numbers according to eq. (12). We have [29]

$$
\begin{gathered}
T_{n n^{\prime}}=\Theta\left[\varepsilon-n^{\prime}\right] \frac{k_{n^{\prime}}}{k_{n}}\left|\delta_{n, n^{\prime}}+f_{n n^{\prime}}^{e}+f_{n n^{\prime}}^{o}\right|^{2} \\
R_{n n^{\prime}}=\Theta\left[\varepsilon-n^{\prime}\right] \frac{k_{n^{\prime}}}{k_{n}}\left|f_{n n^{\prime}}^{e}+f_{n n^{\prime}}^{o}\right|^{2},
\end{gathered}
$$

where $\Theta(x)$ is the Heavyside step-function. The transition probability $W_{n n^{\prime}}$, characterizing the transverse excitation/deexcitation, into a particular channel $n^{\prime}$ from the initial state $n$ is given by the sum of the corresponding transmission and reflection coefficients

$$
W_{n n^{\prime}}=T_{n n^{\prime}}+R_{n n^{\prime}}
$$

Due to the time reversal symmetry of the Hamiltonian we have $T_{n n^{\prime}}=T_{n^{\prime} n}, R_{n n^{\prime}}=R_{n^{\prime} n}$ and $W_{n n^{\prime}}=W_{n^{\prime} n}$. The total transmission (reflection) coefficient $T=\sum_{n^{\prime}} T_{n n^{\prime}}\left(R=\sum_{n^{\prime}} R_{n n^{\prime}}\right)$ is given by the sum of the transmission (reflection) coefficients of all the open channels. Eq.(14) leads to $T+R=1$. 


\section{NUMERICAL APPROACH}

To obtain the observable quantities $T_{n n^{\prime}}, R_{n n^{\prime}}$ and $W_{n n^{\prime}}$ of the scattering process, we have to calculate the matrix elements $f_{n n^{\prime}}$ of the scattering amplitude $\hat{f}$ by matching the numerical solution of the Schrödinger equation (8) with the scattering asymptotics (13). To integrate this multi-channel scattering problem in two dimensions $r$ and $\theta(z$ and $\rho)$ we adopt the discrete-variable method suggested in ref. [30] for solving nonseparable 2D scattering problems. This approach was applied in ref. [31] to the case of a 3D anisotropic scattering problem of ultracold atoms in external laser fields.

First, we discretize the 2D Schrödinger equation (8) on a 2D grid of angular $\left\{\theta_{j}\right\}_{j=1}^{N_{\theta}}$ and radial $\left\{r_{j}\right\}_{j=1}^{N}$ variables. The angular grid points $\theta_{j}$ are defined as the zeroes of the Legendre polynomial $P_{N_{\theta}}(\cos \theta)$ of the order $N_{\theta}$. Using the completeness property of the normalized Legendre polynomials which remains valid also on the chosen angular grid

$$
\sum_{l=0}^{N_{\theta}-1} P_{l}\left(\cos \theta_{j}\right) P_{l}\left(\cos \theta_{j^{\prime}}\right) \sqrt{\lambda_{j} \lambda_{j^{\prime}}}=\delta_{j j^{\prime}},
$$

where $\lambda_{j}$ are the weights of the Gauss quadrature, we expand the solution of equation (8) in the basis $f_{j}(\theta)=\sum_{l=0}^{N_{\theta}-1} P_{l}(\cos \theta)\left(\mathbf{P}^{-1}\right)_{l j}$ according to

$$
\psi(r, \theta)=\frac{1}{r} \sum_{j=1}^{N_{\theta}} f_{j}(\theta) u_{j}(r) .
$$

Here $\mathbf{P}^{-1}$ is the inverse of the $N_{\theta} \times N_{\theta}$ matrix $\mathbf{P}$ with the matrix elements defined as $\mathbf{P}_{j l}=\sqrt{\lambda_{j}} P_{l}\left(\cos \theta_{j}\right)$. Due to this definition one can use the completeness relation $[18)$ in order to determine the matrix elements $\left(\mathbf{P}^{-\mathbf{1}}\right)_{l j}$ as $\left(\mathbf{P}^{-\mathbf{1}}\right)_{l j}=\sqrt{\lambda_{j}} P_{l}\left(\cos \theta_{j}\right)$. It is clear from (19) that the unknown coefficients $u_{j}(r)$ in the expansion are the values $\psi\left(r, \theta_{j}\right)$ of the twodimensional wave function $\psi(r, \theta)$ at the grid points $\theta_{j}$ multiplied by $\sqrt{\lambda_{j}} r$. Near the origin $r \rightarrow 0$ we have $u_{j}(r) \simeq r \rightarrow 0$ due to the definition $(19)$ and the demand for the probability distribution $\left|\psi\left(r, \theta_{j}\right)\right|^{2}$ to be bounded. Substituting (19) into (8) results a system of $N_{\theta}$ Schrödinger-like coupled equations with respect to the $N_{\theta}$-dimensional unknown vector $\mathbf{u}(r)=\left\{\lambda_{j}^{1 / 2} u_{j}(r)\right\}_{1}^{N_{\theta}}$

$$
\left[\mathbf{H}^{(0)}(r)+2(\epsilon \mathbf{I}-\mathbf{V}(r))\right] \mathbf{u}(r)=0
$$

where

$$
\mathbf{H}_{j j^{\prime}}^{(0)}(r)=\frac{d^{2}}{d r^{2}} \delta_{j j^{\prime}}-\frac{1}{r^{2}} \sum_{l=0}^{N_{\theta}-1} \mathbf{P}_{j l} l(l+1)\left(\mathbf{P}^{-1}\right)_{l j^{\prime}}
$$




$$
\mathbf{V}_{j j^{\prime}}(r)=V\left(r, \theta_{j}\right) \delta_{j j^{\prime}}=\left\{V(r)+\frac{1}{2} \omega^{2} \rho_{j}^{2}\right\} \delta_{j j^{\prime}}, \quad \rho_{j}=r \sin \theta_{j},
$$

and $\mathbf{I}$ is the unit matrix. We solve the system of equations 20 on the quasi-uniform radial grid 32

$$
r_{j}=R \frac{e^{\gamma x_{j}}-1}{e^{\gamma}-1}, j=1,2, \ldots, N
$$

of $N$ grid points $\left\{r_{j}\right\}$ defined by mapping $r_{j} \in(0, R \rightarrow+\infty]$ onto the uniform grid $x_{j} \in(0,1]$ with the equidistant distribution $x_{j}-x_{j-1}=1 / N$. By varying $N$ and the parameter $\gamma>0$ one can choose more adequate distributions of the grid points for specific interatomic and confining potentials.

By mapping the initial variable $r$ in Eq. 20 onto $x$ we obtain

$$
\left[\mathbb{H}^{(0)}(x)+2\{\epsilon \mathbf{I}-\mathbf{V}(r(x))\}\right] \mathbf{u}(r(x))=0
$$

with

$$
\mathbb{H}_{j j^{\prime}}^{(0)}(x)=f^{2}(x) \delta_{j j^{\prime}}\left(\frac{d^{2}}{d x^{2}}-\gamma \frac{d}{d x}\right)-\frac{1}{r^{2}(x)} \sum_{l=0}^{N_{\theta}-1} \mathbf{P}_{j l} l(l+1)\left(\mathbf{P}^{-1}\right)_{l j^{\prime}}
$$

where

$$
f(x)=\frac{e^{\gamma}-1}{R e^{\gamma x} \gamma}
$$

The uniform grid with respect to $x$ gives 6-order accuracy for applying a 7-point finitedifference approximation of the derivatives in the equation (24). Thus, after the finitedifference approximation the initial 2D Schrödinger equation (8) is reduced to the system of $\mathrm{N}$ algebraic matrix equations

$$
\begin{aligned}
& \sum_{p=1}^{3} \mathbb{A}_{j-p}^{j} \mathbf{u}_{j-p}+\left[\mathbb{A}_{j}^{j}+2\left\{\epsilon \mathbf{I}-\mathbf{V}_{j}\right\}\right] \mathbf{u}_{j}+\sum_{p=1}^{3} \mathbb{A}_{j+p}^{j} \mathbf{u}_{j+p}=0, j=1,2, \ldots, N-3 \\
& \mathbf{u}_{j}+\alpha_{j}^{(1)} \mathbf{u}_{j-1}+\alpha_{j}^{(2)} \mathbf{u}_{j-2}+\alpha_{j}^{(3)} \mathbf{u}_{j-3}+\alpha_{j}^{(4)} \mathbf{u}_{j-4}=\mathbf{g}_{j} \quad j=N-2, N-1, N
\end{aligned}
$$

where each coefficient $\mathbb{A}_{j^{\prime}}^{j}$ is a $N_{\theta} \times N_{\theta}$ matrix, each $\alpha_{j}$ is a diagonal $N_{\theta} \times N_{\theta}$ matrix and each $\mathbf{g}_{j}$ is a $N_{\theta^{-}}$-dimensional vector. Here the functions $\mathbf{u}_{-3}, \mathbf{u}_{-2}, \mathbf{u}_{-1}$ and $\mathbf{u}_{0}$ in the first three equations of the system (for $j=1,2$ and 3) are eliminated by using the "left-side" boundary conditions: $\mathbf{u}_{0}=0$ and $\mathbf{u}_{-j}=\mathbf{u}_{j}(j=1,2,3)$. The last three equations in this system for $j=N, N-1$ and $N-2$ are the "right-side" boundary conditions approximating at the edge points $r_{N-2}, r_{N-1}$ and $r_{N}=R$ of the radial grid, the scattering asymptotics (13) for the desired wave function $\mathbf{u}\left(r_{j}\right)$. In order to construct the "right-side" boundary 
conditions (27) at $j=N-2, N-1$ and $N$ we used an idea of ref.[31] i.e. the asymptotic behaviour (13) at the edge points $r_{N-2}, r_{N-1}$ and $r_{N}=R$ are considered as a system of vector equations with respect to the unknown vector $f_{n n^{\prime}}$ of the scattering amplitude for a fixed $n$. By eliminating the unknowns $f_{n n^{\prime}}$ from this system we implement the "right-side" boundary conditions defined by Eqs. 27) at $j=N-2, N-1$ and $N$ (see Appendix A).

The reduction of the $2 \mathrm{D}$ multi-channel scattering problem to the finite-difference boundary value problem (27) permits one to apply efficient computational methods. Here we use, in the spirit of the $L U$-decomposition [33], and the sweep method [34] (or the Thomas algorithm [35]), a fast implicit matrix algorithm which is briefly described in Appendix B. The block-diagonal structure of the matrix of the coefficients in the system of equations(27) with the width of the diagonal band equal to $7 \times N_{\theta}$ makes this computational scheme an efficient one.

Solving the problem (27) for the defined initial vector $k_{n}$ and a fixed $n$ from the possible set $0 \leq n \leq n_{e}$ we first calculate the vector function $\psi\left(k_{n}, r, \theta_{j}\right)$. Then, by matching the calculated vector $\psi\left(k_{n}, R, \theta_{j}\right)$ with the asymptotic behaviour (13) at $r=R$, we calculate the $n$-th row of the scattering amplitude matrix $f_{n n^{\prime}}$ describing all possible transitions $n \rightarrow n^{\prime}=$ $0,1, \ldots, n_{e}$. This procedure is repeated for the next $n$ from $0 \leq n \leq n_{e}$. After calculating all the elements $f_{n n^{\prime}}$ of the scattering amplitude we obtain any desired scattering parameter $T, R$ or $W$.

\section{RESULTS AND DISCUSSION}

With the above-described method being implemented we have analyzed the two-body scattering under the transverse harmonic confinement for both cases of identical and distinguishable colliding atoms. For confined scattering of identical atoms one has to distinguish the bosonic and fermionic cases. In the case of two colliding bosons the two-body wave function must be symmetric and only even scattering amplitude provides us with a nonzero contribution. First, we show that our result for the special case $\varepsilon<1$ of a single-channel scattering is in agreement at $\varepsilon \rightarrow 0$ with the s-wave pseudopotential approach [13], and, particularly, reproduces s-wave CIR predicted and analyzed in refs. [13, 14, 24, 26, 29] for bosons. Then we extend our consideration to the multi-channel scattering $\varepsilon>1$. We demonstrate that our results are in a good agreement in the limit of a long-wavelength trap 
$\omega=2 \pi c / \lambda \rightarrow 0$ with the analytical expression given in [29] which has been obtained in the s-wave pseudopotential approach for the zero-energy limit. The range of validity of the analytical investigation in ref. [29] is explored. Next we present results for multi-channel scattering of two fermions under transverse harmonic confinement. For a fermionic collision the two-body wave function is antisymmetric, i.e. only the odd scattering amplitude is nonzero. In the special case of single-channel scattering we reproduce the p-wave CIR for fermions [18]. These results are also in agreement with our previous investigations in refs. [24, 25, 26] performed within a wave-packet propagation method [26]. Finally we consider the confined multi-channel scattering of two distinguishable atoms. In this case both even and odd amplitudes contribute to the scattering process.

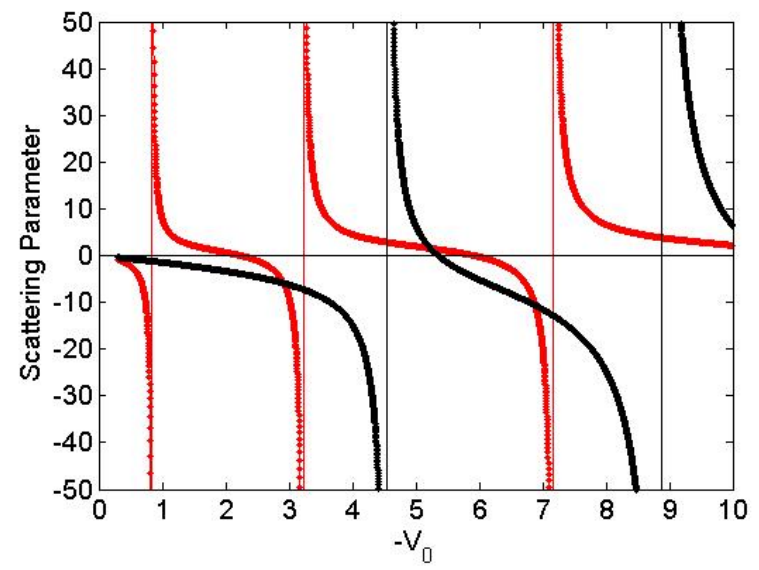

FIG. 1: s- and p-wave scattering parameters $a_{s}$ (red) and $V_{p}$ (black) as a function of the potential depth $V_{0}$ for the free-space effective potential $V(r)+l(l+1) /\left(2 r^{2}\right)$. Divergences correspond to the appearance of new bound (s-wave scattering) or shape resonant (p-wave scattering) states in the effective potential. All quantities are given in the units (7).

For modeling different interatomic interactions in the subsequent sections we vary the depth $V_{0}$ of the potential (6) in the wide range $-10<V_{0}<0$ for a fixed width $r_{0}=1$. In free space, this potential being superimposed with the centrifugal term $l(l+1) /\left(2 r^{2}\right)$ makes an effective potential which may support some even or odd bound states depending on the value of the quantum number $l$ and the parameter $V_{0}$. For $l \neq 0$ there might be also some shape resonances for certain relative energies. These shape resonances may enhance strongly the contribution of $l \neq 0$ partial waves in the energy domain where one would have expected a pure $l=0$ scattering. 
It is known that in the zero-energy limit, when the scattering process does not depend on the details of the potential, the collision can be described by a single parameter: the s-wave scattering length $a_{s}=-\lim _{k \rightarrow 0} \tan \delta_{s}(k) / k$ for $l=0$ (bosonic collision) and the p-wave scattering volume $V_{p}=-\lim _{k \rightarrow 0} \tan \delta_{p}(k) / k^{3}$ for $l=1$ (fermionic collision). For sufficiently low collision energy the contribution of the partial waves with larger $l$ can be neglected. In Fig, 11 we have plotted $a_{s}$ and $V_{p}$ in the region $-10<V_{0}<0$. Fig.1 demonstrates the rich spectral structure of the chosen form of the interatomic interaction: the scattering parameters $a_{s}$ and $V_{p}$ can be positive or negative and they diverge for the values of $V_{0}$ corresponding to the appearance of new bound states. In the case of p-wave scattering the increase of the depth $V_{0}$ of the potential first leads to a shape resonance which approaches zero energy and finally transforms to a p-wave bound state.

\section{A. Multi-channel scattering of bosons}

For two bosons colliding in a transverse harmonic confinement the scattering wave function is symmetric with respect to the exchange $z \rightarrow-z$, i.e. $f_{n n^{\prime}}^{o}=0$ in Eq. (13). We consider multi-channel scattering with the dimensionless energy $\varepsilon=\epsilon /(2 \omega)-1 / 2<4(12)$ permitting the collisional transverse excitations/deexcitations $n \rightarrow n^{\prime}<4$ up to four open channels (four-mode regime). For comparison with analytical results [14, 29] obtained in the s-wave pseudopotential approach, we have extracted the effective quasi-1D coupling constant $g_{1 D}=\lim _{k \rightarrow 0} \operatorname{Re}\left\{f_{00}^{e}(k)\right\} / \operatorname{Im}\left\{f_{00}^{e}(k)\right\} k / \mu$ as well as the transmission coefficient $T=T_{00}(15$ and the scattering amplitude $f_{00}^{e}(13)$, as a function of the scattering length $a_{s}$ in the singlemode regime $(0<\varepsilon<1)$. The calculated parameters are presented along with the analytical results in Fig,2 for $\omega=0.002$ and the longitudinal relative energy $\epsilon_{\|}=\epsilon-\epsilon_{\perp}=0.0002$. Fig,2(a) shows the coupling constant $g_{1 D}$ as a function of the scattering length $a_{s}$. Our numerical result clearly exhibit a singularity at $a_{s} / a_{\perp} \approx 1 / C$ with $C=-\zeta(1 / 2)=1.4603 .$. , which corresponds to the well-known s-wave CIR [13, 14]. In Fig,2(b) we present the transmission coefficient $T$ versus $a_{s}$. The transmission coefficient $T$ goes to unity (total transmission) when $a_{s}$ tends to zero (i.e. no interaction between the atoms), while at the CIR position, it exhibits the well-known minimum (blocking of the atomic current by the CIR). Fig.2(c) shows the scattering amplitude $f_{00}^{e}$ as a function of $a_{s}$. The amplitude approaches zero at $a_{s} / a_{\perp}=0$ and -1 at the CIR position, which results in total transmission and total 
reflection respectively. In general the presented values of $f_{00}^{e}$ are in very good agreement with the analytical results.
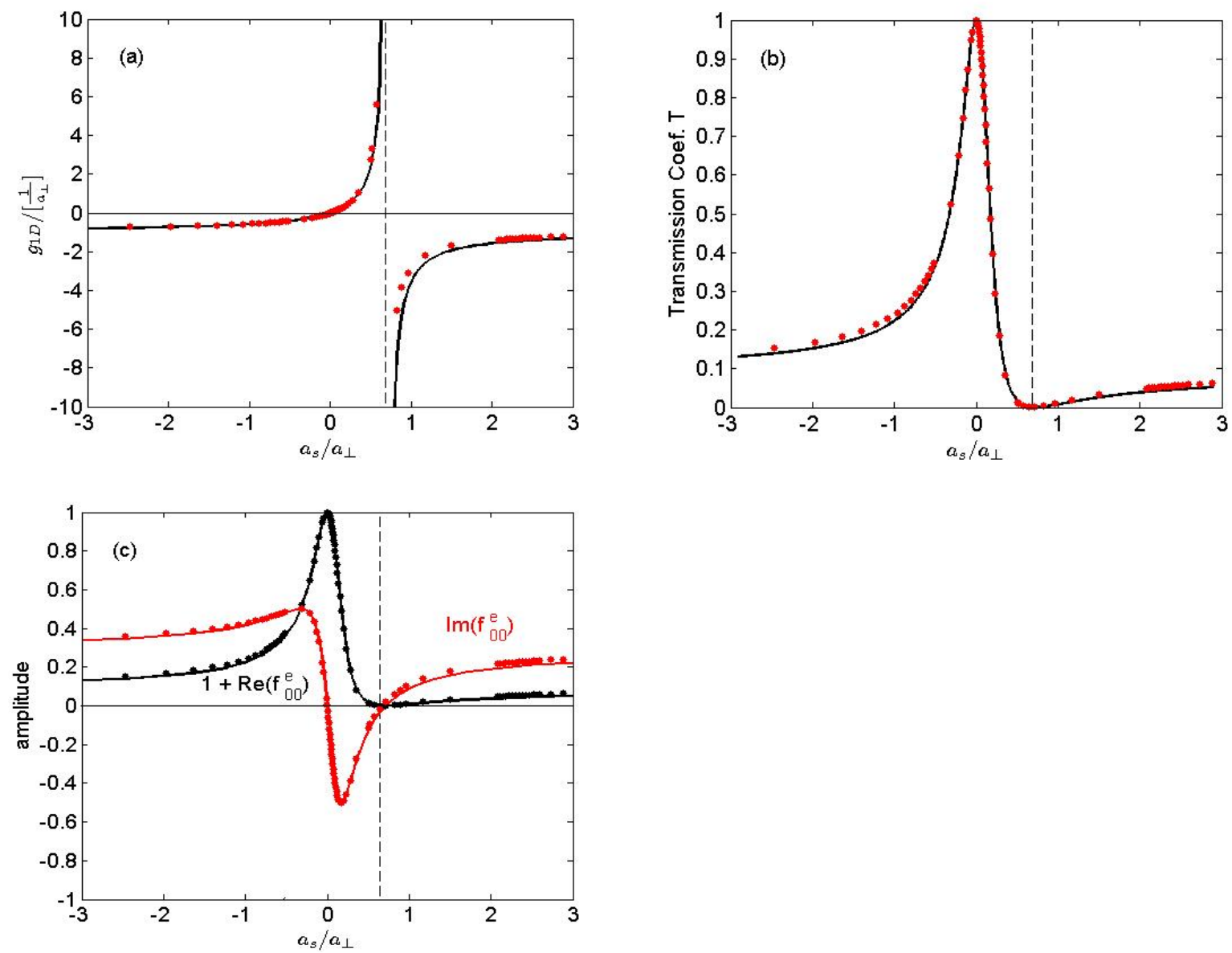

FIG. 2: (a) The effective quasi-1D coupling constant $g_{1 D}$, (b) the transmission coefficient $T$ and (c) the scattering amplitude $f_{00}^{e}$ as a function of the scattering length $a_{s}$ for one-channel scattering of two bosons under a harmonic confining potential with $\omega=0.002$ for a longitudinal relative energy $\epsilon_{\|}=0.0002$ together with the analytical results obtained for the s-wave pseudo-potential zeroenergy limit(solid curves ) [13, 14, 29]. The constant $V_{0}$ is varied in the region $-2.30<V_{0}<-0.32$.

In the multi-mode regime our results are in good agreement with the analytical ones obtained within the s-wave pseudopotential approach in the zero-energy limit[29] for longwavelength traps (tight confinement: $\omega=2 \pi c / \lambda \rightarrow 0$ ). In Fig. 3 we present our scattering amplitude $f_{00}^{e}$ along with the analytical results as a function of the dimensionless energy $\varepsilon$ for $\omega=0.0002$ and $V_{0}=-3.0$. Apart from energies close to the channel thresholds, the real and imaginary part of the scattering amplitude $f_{00}^{e}$ show a monotonous behaviour: $\operatorname{Re}\left(f_{00}^{e}\right)$ is monotonically increasing and approaching zero asymptotically whereas $\operatorname{Im}\left(f_{00}^{e}\right)$ 
decays monotonically and also approaches zero for large values of $\varepsilon$. The peak structure located at integer values of $\varepsilon$ is due to the resonant scattering once a new previously closed channel opens with increasing energy. There is a good agreement between our results and the analytical ones given by Eq.(6.9) in ref. [29] for the complete range $0<\varepsilon<4$. However, we encounter major deviations with increasing $\omega$ except for narrow regions close to the channel thresholds (as $\varepsilon$ approaches integer values) for the real parts of the scattering amplitudes (see Fig.4). These deviations are most presumably due to the energy dependence of the s-wave scattering length which is neglected in ref. [29].
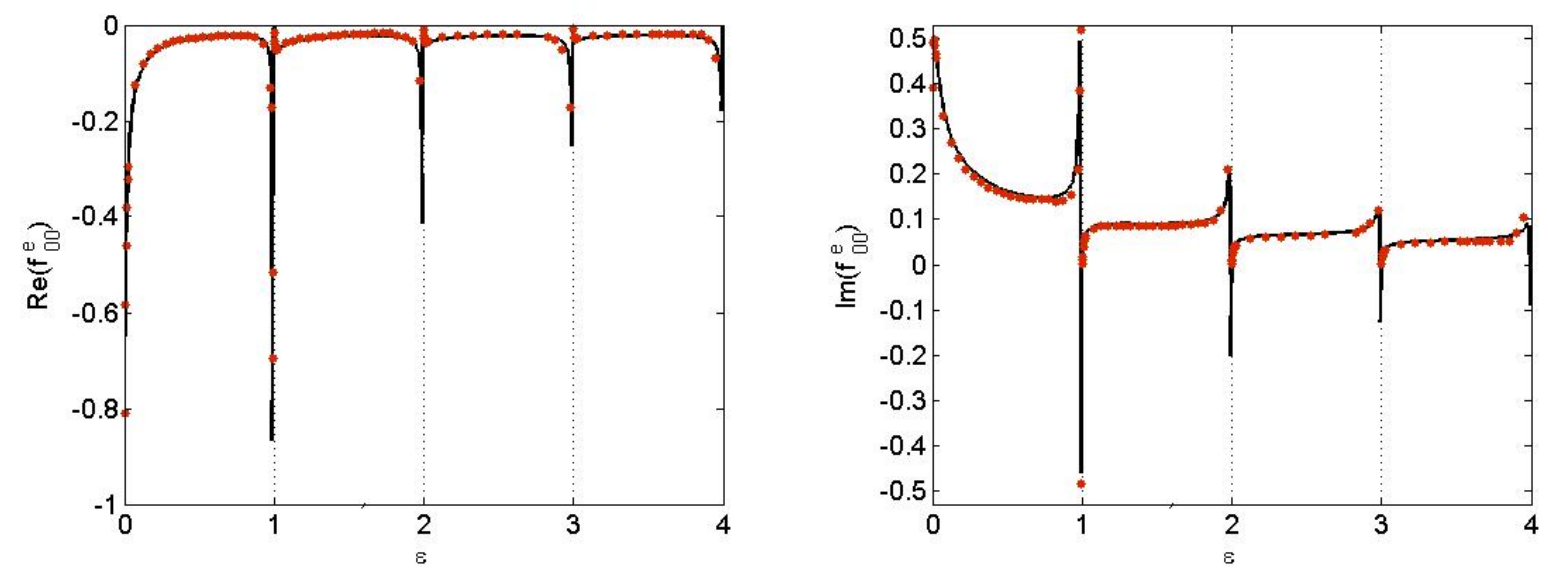

FIG. 3: The scattering amplitude $f_{00}^{e}$ (dots) as a function of the dimensionless energy $\varepsilon$ along with the analytical results (solid curves) for $\omega=0.0002$ and $V_{0}=-3.0\left(a_{s}=-8.95\right)$.

For the multi-channel regime $(\varepsilon>1)$ we find a strong dependence of the total transmission coefficient $T$ on the population of the initial state $n$, except for the case $a_{s} / a_{\perp} \rightarrow 0$ of noninteracting bosons in free space. Fig 5 shows the calculated transmission coefficients 15 as a function of $a_{s} / a_{\perp}$ for $\omega=0.002$ for the (a) two-mode regime with $\varepsilon=1.05$ and (b) the three-mode regime with $\varepsilon=2.05$. Note, that all these cases correspond to near-threshold collision energies if the maximal integer is subracted from $\varepsilon$. Similar to the single-mode regime, $T$ goes to unity (total transmission) when $a_{s}$ tends to zero. We also encounter a minimum. However, the value of the transmission at the minimum is not zero anymore in the multi-mode regime, the larger the number of open channels, the position of the minima will be more shifted to the left. For a fixed value of the ratio $a_{s} / a_{\perp} \neq 0$, a lower initially populated transverse level $n$, leads to a larger total transmission.

In Fig. 6 we present the transmission as a function of the dimensionless energy $\varepsilon$ for the 

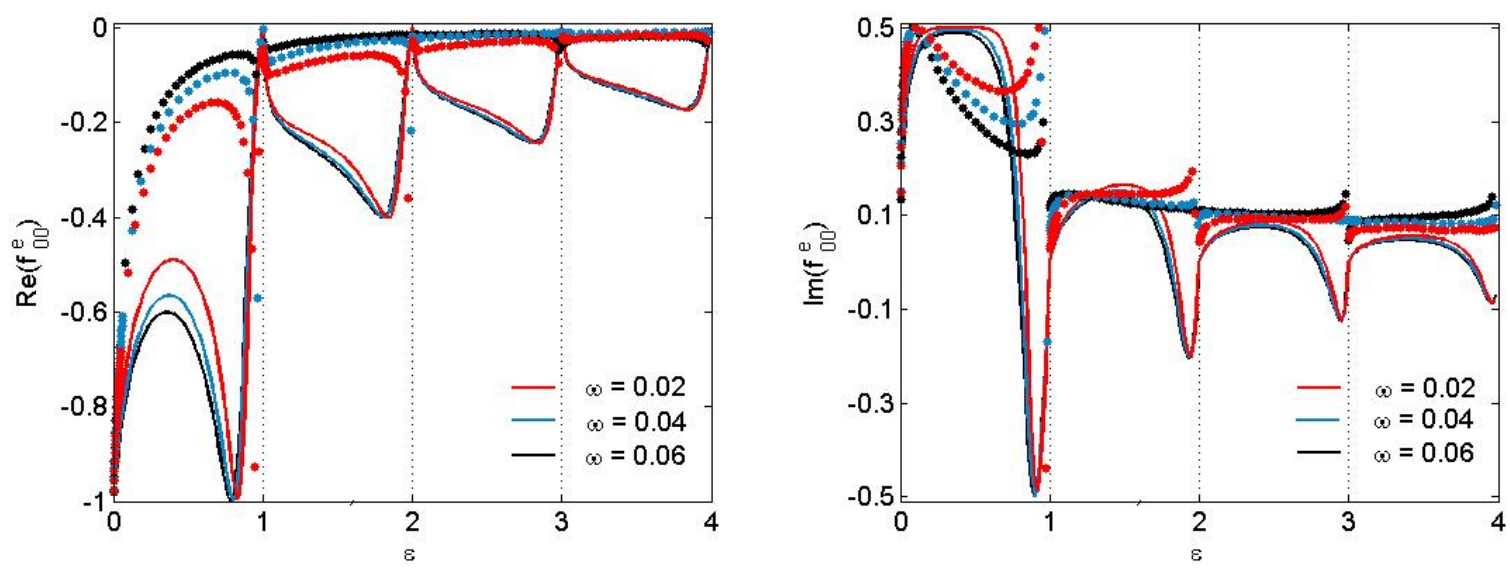

FIG. 4: The scattering amplitude $f_{00}^{e}$ (dots) as a function of the dimensionless energy $\varepsilon$ along with the analytical results (solid curves) for $\omega=0.02$ (red), $\omega=0.04$ (blue) and $\omega=0.06$ (black). The amplitudes have been calculated for $V_{0}=-3.0\left(a_{s}=-8.95\right)$.
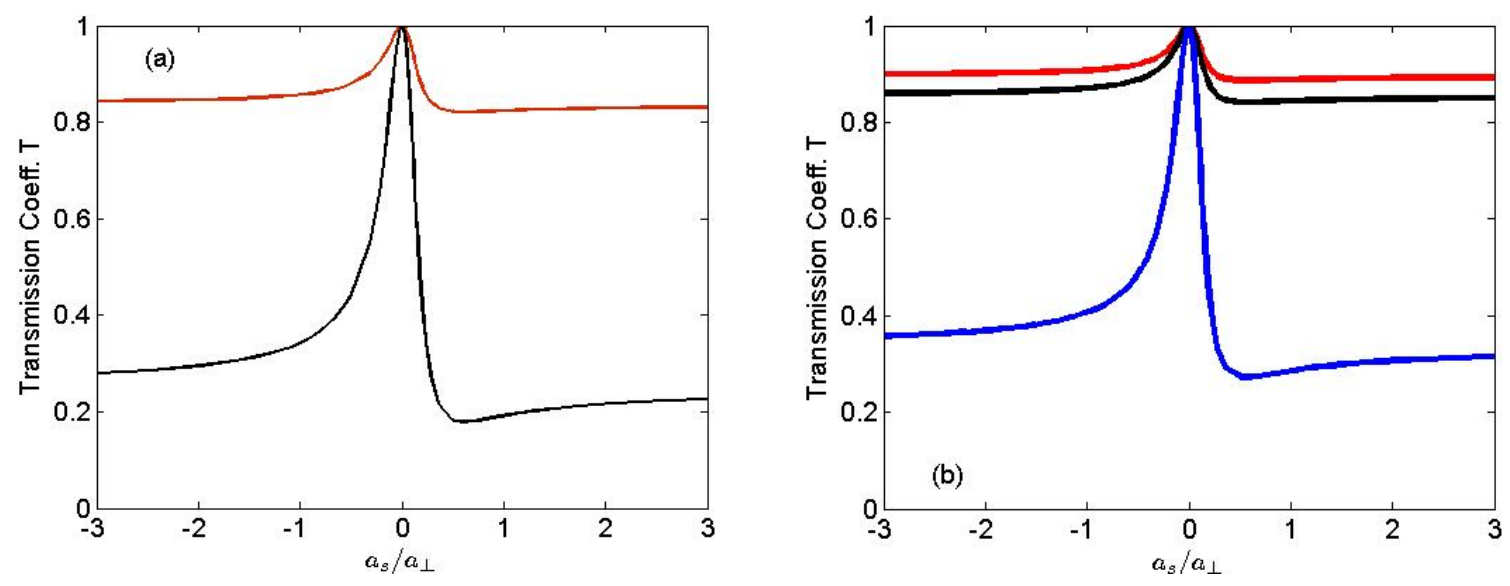

FIG. 5: The total transmission coefficient $T$ for a bosonic collision in a harmonic confinement $\omega=0.002$ as a function of $a_{s} / a_{\perp}$ for (a) two-open channels with $\varepsilon=1.05$ being initially in the transverse ground state $n=0$ (red) and first excited state $n=1$ (black), and (b) three-open channels with $\varepsilon=2.05$ being initially in the ground state $n=0$ (red), first excited state $n=1$ (black) and second excited state $n=2$ (blue).

two cases of the system being initially in the ground $n=0$ (a) or first excited $n=1$ (b) transverse states, for different values of the ratio $a_{s} / a_{\perp}$. With increasing collision energy the transmission coefficient exhibits a nonmonotonic behaviour: we observe a sequence of minima and peaks. For $0<\varepsilon<1$ the value of $T$ at the minimum is zero. As the number of open channels increases with increasing collision energy $\epsilon$, the transmission values at the 
corresponding minima increase strongly. The corresponding transmission peaks $T=1$ are located at the channel thresholds. The shape of the transmission 'valleys' in between two integer values of $\varepsilon$ as well as the positions of the minima strongly depend on the ratio $a_{s} / a_{\perp}$ which can be changed by varying the strength of the interatomic interaction $V_{0}$ or the trap frequency $\omega$. Both, changing $V_{0}$ and/or $\omega$ leads to energetical shifts of the bound states (in particular for the excited transversal channels) of the atoms in the presence of the confinement. If the collision energy coincides with a bound state of the corresponding closed channel we encounter an occupation of the closed channel in the course of the scattering process, i.e. a Feshbach-resonance occurs. The interpretation of the minimum of the transmission $T$ in terms of a Feshbach-resonance at the point $a_{s} / a_{\perp}=1 / C$ for the zero-energy limit was provided in refs. [13, 14], where it was shown that the origin of the CIR is an intermediate occupation of a bound state belonging to an excited transverse (closed) channel.

To demonstrate that the above-discussed behaviour (minima) of the transmission coefficient $T(\varepsilon)$ in certain regions of $\varepsilon$ is due to Feshbach resonances we have analyzed the probability density of the scattering wave function of the atoms in the trap. In the singlemode regime and in the zero-energy limit we encounter the well-known CIR: Fig 7 shows the corresponding probability density $|\psi(x, z)|^{2}$ for an initial transverse ground state $n=0$ and $\varepsilon=0.05$ as well as $a_{s} / a_{\perp}=0.68$. For small $|z|$ one observes additional two pronounced peaks along the transverse $(x-)$ direction corresponding to the occupation of the bound state (with the binding energy $\varepsilon_{n=1}^{B} \sim 0$ ) in the first excited closed channel of the transverse potential. The probability density tends to zero as $z \rightarrow+\infty$. This leads to a zero of the transmission $T(\varepsilon)$ for $\varepsilon \rightarrow 0$ (see Fig $6($ a) ) corresponding to the zero-energy CIR.

In Fig 8 we show the probability densities $|\psi(x, z)|^{2}$ at $a_{s} / a_{\perp}=+4.39$ for several values of the dimensionless energy $\varepsilon$ for (a) the single- and (b) two-mode regimes for collisions with initial transverse state $n=0$. The corresponding probability density exhibits for small values of $|z|$ additional two (for the single-mode regime) and four (for the two-mode regime) pronounced peaks with respect to the transverse $(x$-)direction as $\varepsilon$ approaches the CIRposition. This demonstrates the occupation of bound states of higher, namely first excited (with binding energy $\varepsilon_{n=1}^{B}$ ) and second excited (with binding energy $\varepsilon_{n=2}^{B}$ ) channels in the course of the scattering process. The corresponding transmission values are also indicated in Fig. 8. Zero transmission is observed also for $a_{s} / a_{\perp}=+4.39$ and $\varepsilon=0.75$ with no probability density being present for large positive values of $z$, see Fig,8(a). 

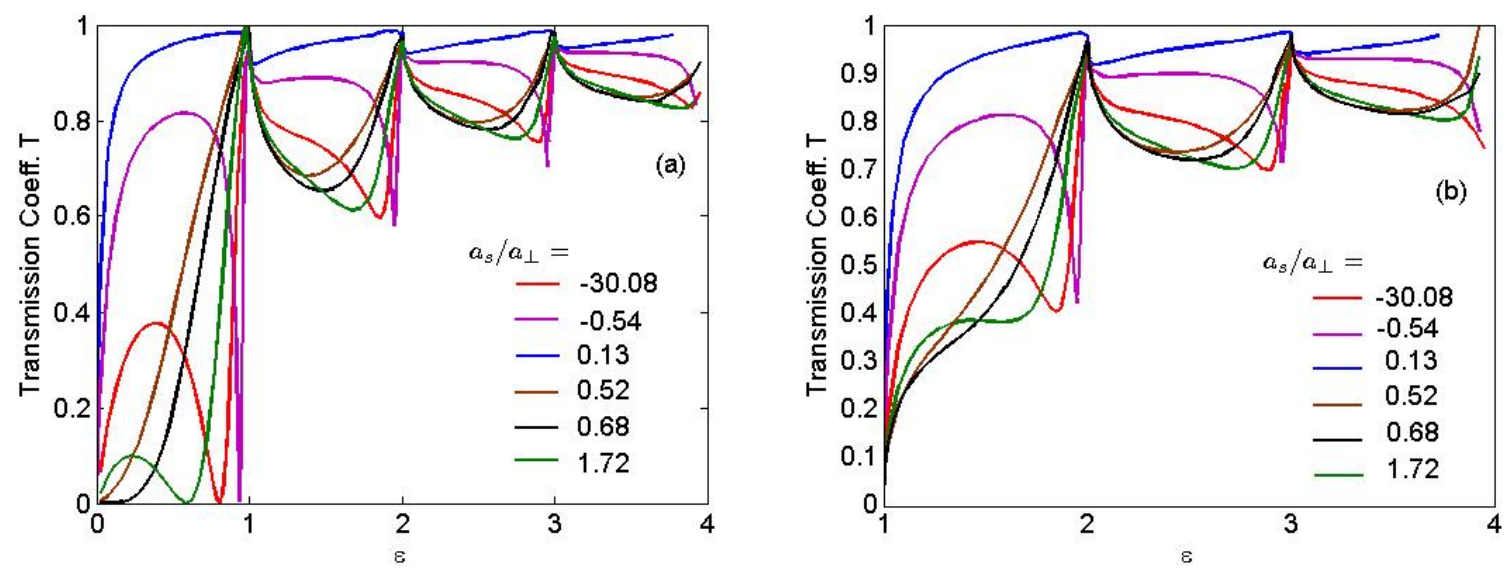

FIG. 6: The total transmission coefficients $T$ for bosonic collisions as a function of the dimensionless energy $\varepsilon$ for the two cases of the system being initially in the ground $n=0$ (a) and first excited $n=1$ (b) transverse states, for several ratios of $a_{s} / a_{\perp}$ and $\omega=0.002$. The black curve corresponds to $a_{s} / a_{\perp}=1 / C$ for which the zero-energy CIR in the single-mode regime is encountered.

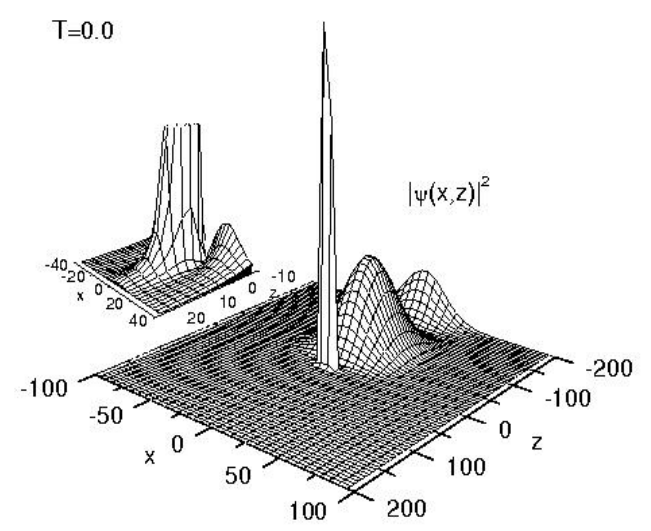

FIG. 7: The probability density $|\psi(x, z)|^{2}$ for bosonic collisions as a function of $x$ and $z$ at $a_{s} / a_{\perp}=$ 0.68 for $\varepsilon=0.05$ - zero-energy CIR . The corresponding transmission values are also indicated. The result has been obtained for $\omega=0.002$.

The energy of the bound state $\varepsilon_{n=1}^{B}$, that leads to a minimal transmission due to a resonant scattering process, changes with varying $a_{s} / a_{\perp}$ as follows. It is below $\varepsilon=0$ for $0<a_{s} / a_{\perp}<0.68$ and consequently no minimum is encountered for $T(\varepsilon)$ in the range $0<\varepsilon<1$ (see fig. 6). At the position of the zero-energy CIR it is located just above the threshold $\varepsilon=0$ leading to a zero transmission for $\varepsilon \rightarrow 0$. For $a_{s} / a_{\perp}>0.68$ the bound state energy is somewhere in between the channel thresholds $\varepsilon=0$ and $\varepsilon=1$ whereas for 

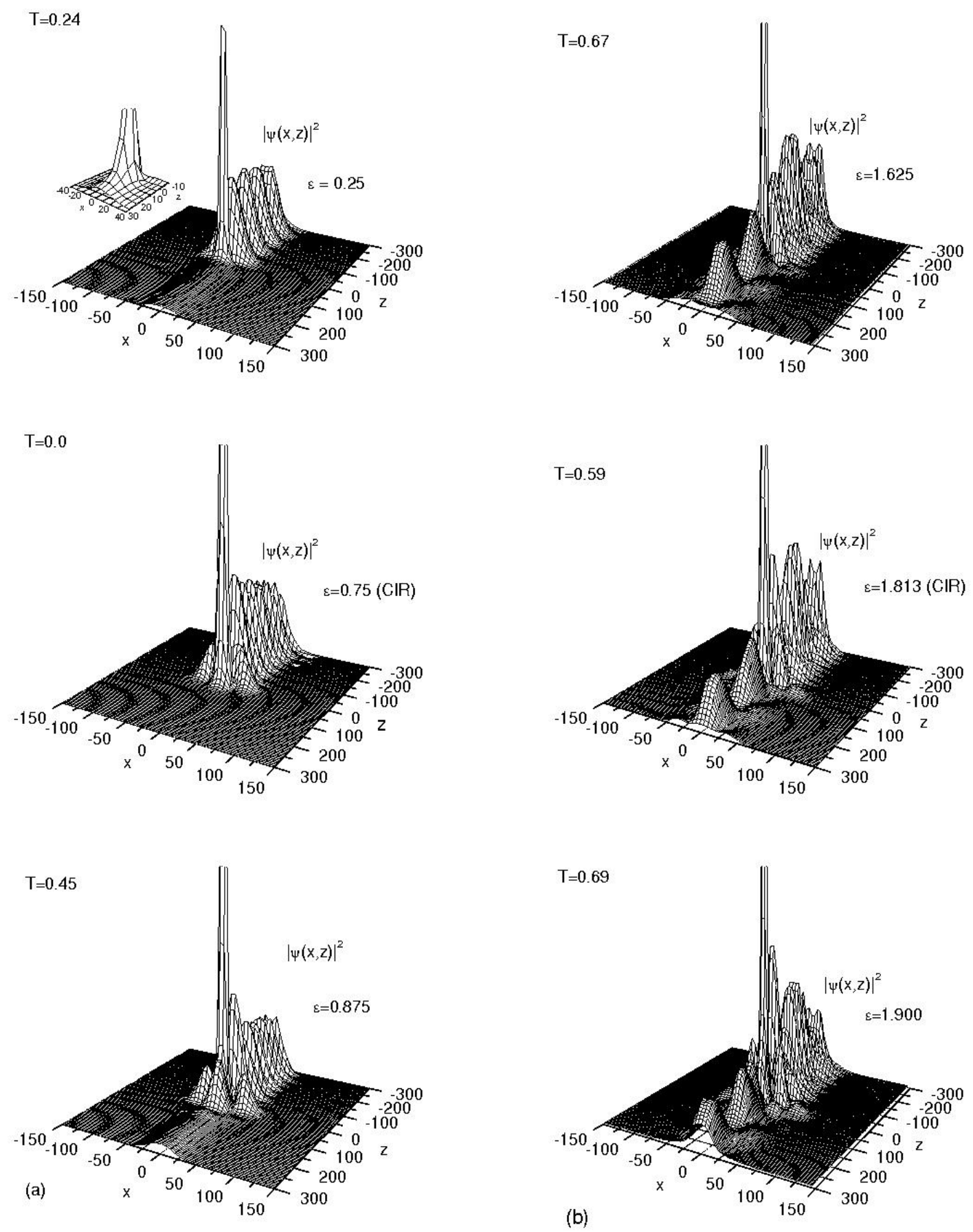

FIG. 8: The probability density $|\psi(x, z)|^{2}$ for bosonic collisions as a function of $x$ and $z$ at $a_{s} / a_{\perp}=$ +4.39 for two cases of the single-mode regime (a) and two-mode regime (b) with different values of $\varepsilon$. The corresponding transmission values are also indicated. All subfigures are for $\omega=0.002$ and $n=0$. 
$a_{s} / a_{\perp}<0$ it is below but close to $\varepsilon=1$ leading again to a corresponding minimum of $T$. For both cases, $a_{s} / a_{\perp}<0$ and $a_{s} / a_{\perp}>0.68$, an increase of $a_{s} / a_{\perp}$ leads to a narrow transmission well and the corresponding transmission minimum is shifted towards the next higher channel threshold. The dependence of $\varepsilon_{n=1}^{B}$ on the parameter $a_{s} / a_{\perp}$ is in agreement with the pseudopotential analysis given in ref.[14] (see Fig. 2 of this ref.).

In Fig 9 (a) we show the transition probabilities $W_{n n^{\prime}}$ as a function of $a_{s} / a_{\perp}$ for $\omega=0.002$ and $\varepsilon=3.05$ corresponding to four open channels. We observe that the probability of remaining at the same initial state, $W_{n n}$ (i.e. elastic scattering) is in the complete range of the ratio $a_{s} / a_{\perp}$ much larger than the probability of a transition into a different state, $W_{n n^{\prime}}$ (i.e. inelastic scattering). With increasing $n$ or $n^{\prime}$ the inelastic transition probabilities $W_{n n^{\prime}}$ increase but the elastic probabilities $W_{n n}$ decrease. In an inelastic (elastic) collision $W_{n n^{\prime}}\left(W_{n n}\right)$ goes to zero (unity) as $a_{s}$ tends to zero. $W_{n n^{\prime}}\left(W_{n n}\right)$ possess a maximum (minimum) at the resonance position $a_{s} / a_{\perp} \approx 0.35$ consistent with the minimum of $T(\varepsilon)$ at $\varepsilon=3.05$. It is instructive to see how the distribution of the initial flux among the open channels changes due to pair collisions as a function of the collision energy. Fig 9 (b) shows the transition probabilities $W_{n n^{\prime}}$ as a function of the dimensionless energy up to four open channels. The probability of elastic scattering remains larger than that of inelastic scattering in the complete range of the energy. For two open channels the elastic collision probability $W_{n n}$ is independent of the initial state $\left(W_{00}=W_{11}\right)$. For a higher number of open channels $W_{n n}$ is decreasing with increasing initial value of $n$. Near the thresholds, the probabilities of the inelastic (elastic) transitions $W_{n n^{\prime}}\left(W_{n n}\right)$ go to zero (unity).

\section{B. Multi-channel scattering of fermions}

In this section we focus on fermionic collisions in harmonic traps. In this case the interatomic wave function is anti-symmetric with respect to the interchange of the two fermions and the even amplitude $f_{n n^{\prime}}^{e}$ in eqs.(13) is zero. We have analyzed the multichannel scattering of fermions up to four open transverse channels for different interatomic interactions, by varying the potential strength $V_{0}$ in the vicinity of the value $V_{0}=-4.54$ (see Fig.1) generating a resonant p-wave state in free space. Fig. 10 shows corresponding results for the single-mode regime. Fig. 10(a) shows the mapped coupling constant $g_{1 D}^{\text {map }}=\lim _{k \rightarrow 0} \operatorname{Im}\left\{f^{o}(k)\right\} / \operatorname{Re}\left\{f^{o}(k)\right\} \mu k[18,26]$ as a function of the p-wave scattering length 

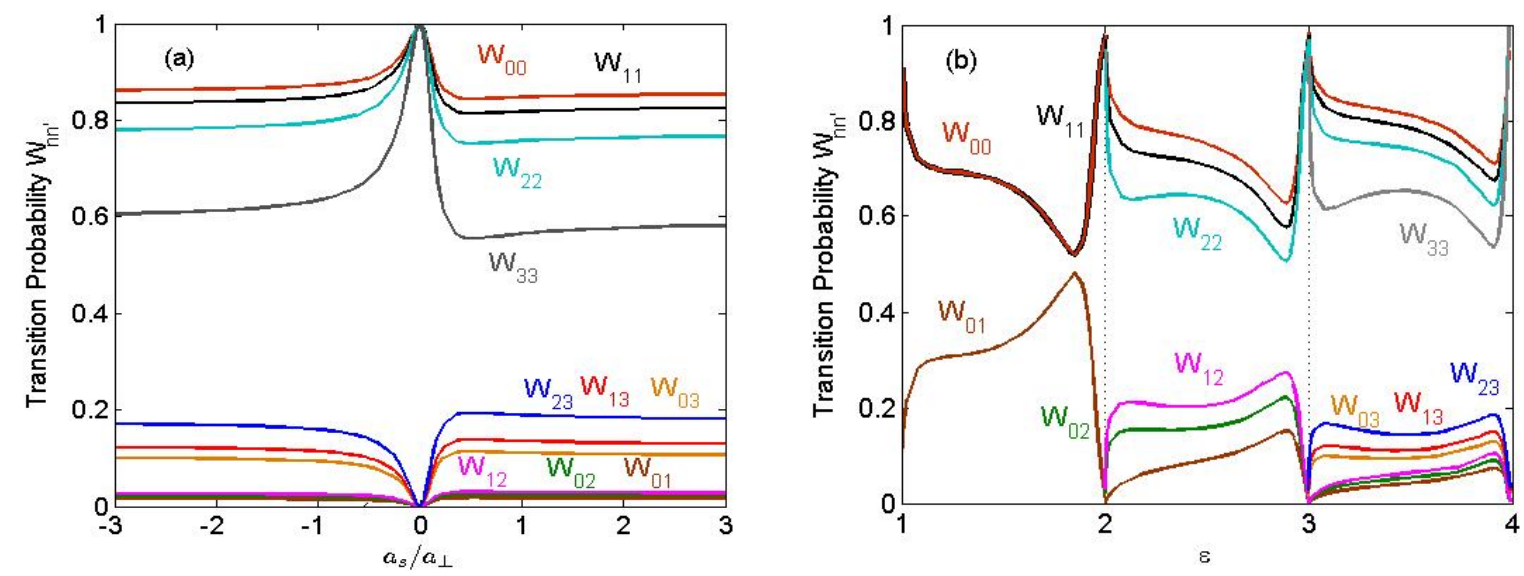

FIG. 9: (a) The transition probabilities $W_{n n^{\prime}}$ as a function of $a_{s} / a_{\perp}$ in the four mode regime for $\varepsilon=3.05$. (b) The calculated transition probabilities $W_{n n^{\prime}}$ as a function of $\varepsilon$ up to four open channels for $a_{s} / a_{\perp}=-30.08$. $\omega=0.002$ for both subfigures.

$a_{p}=\sqrt[3]{V_{p}}$. The mapped coupling constant $g_{1 D}^{\text {map }}$ goes to zero at the position of the mapped CIR [18], which for $\omega=0.002$ and $\epsilon_{\|}=0.0002$ is equal to $a_{p} / a_{\perp}=-0.31$. The position of the mapped CIR obviously depends on the values of $\omega$ and $\varepsilon$. In Fig.10(b) we have plotted the transmission coefficient $T$ as a function of $a_{p}$. The transmission coefficient exhibits a minimum and an accompanying well (i.e. the blocking due to the resonance) at the position of the CIR, and tends to unity (i.e. total transmission) for $a_{p}$ far from the CIR-position. For larger $\omega$ the well becomes wider, and its minimum is shifted to the left, see also ref. [24]. Fig. 10(c) shows the scattering amplitude $f_{00}^{o}$ as a function of $a_{p}$ for $\omega=0.002$ and $\epsilon_{\|}=0.0002$. The amplitude approaches zero far from the CIR position and -1 at the CIR position , which results in total transmission, $T=\left|1+f_{00}^{o}\right|^{2} \rightarrow 1$ (i.e. the fermions do not scatter each other) and total reflection, $T=\left|1+f_{00}^{o}\right|^{2} \rightarrow 0$ (i.e. strongly interacting and impenetrable fermions), respectively.

In Fig. 11 we present the total transmission coefficient as a function of $a_{p}$ in the two-mode regime for $\varepsilon=1.05$ (a) and three-mode regime for $\varepsilon=2.05$ (b) for $\omega=0.002$. Similar to the single-mode regime, $T$ exhibits a minimum and accompanying well, however the position of the minimum is shifted to the right and the value at the minimum is nonzero. For the lower degree of transversal excitation, we observe a deeper and narrower transmission well. With increasing energy the transmission well becomes wider and more shallow and its position is shifted to larger values of $a_{p} / a_{\perp}$. This is also demonstrated in Fig.12, where the transmission 

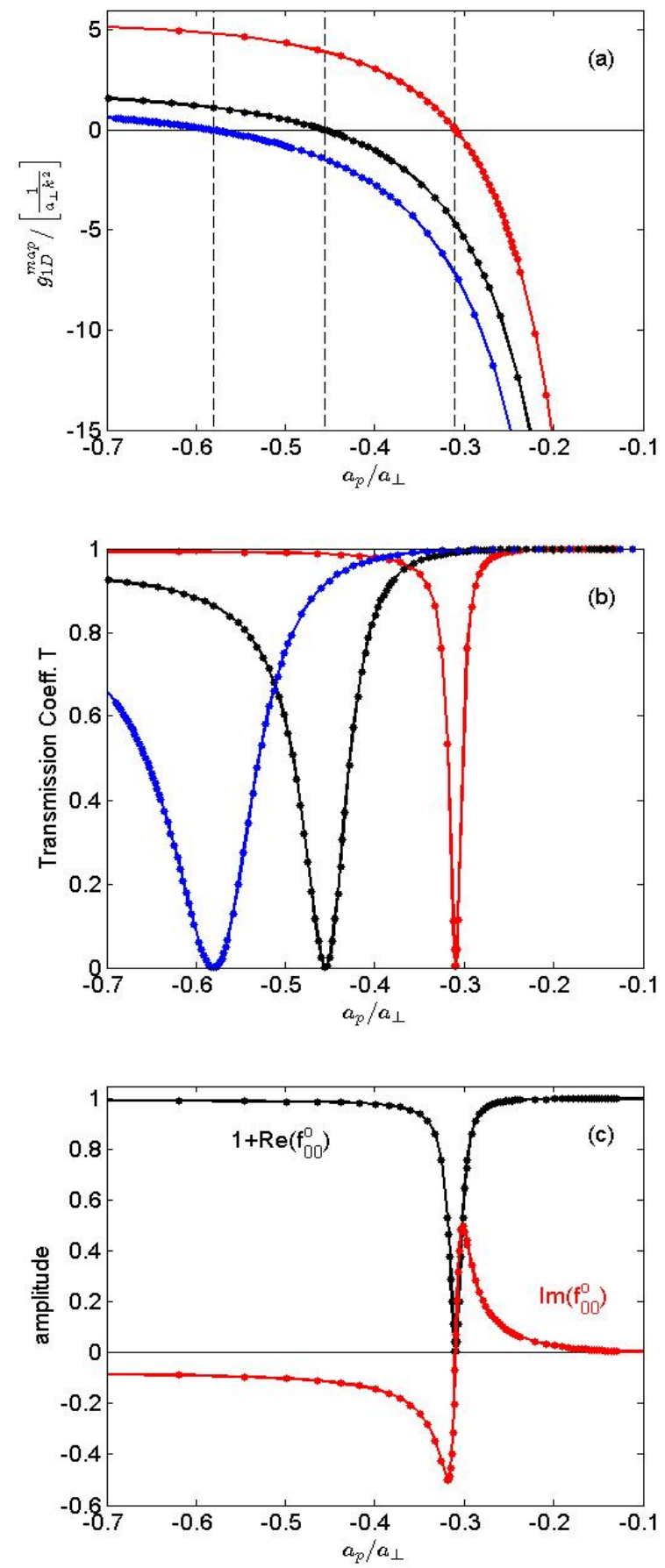

FIG. 10: (a-b) The mapped coupling constant $g_{1 D}^{\text {map }}$ and the transmission coefficient $T$ as a function of the scattering length $a_{p}$ for single-channel scattering of two fermions under a harmonic confinement $\omega=0.002$ and for the longitudinal energy $\epsilon_{\|}=0.0002$ (red), $\omega=0.02, \epsilon_{\|}=0.002$ (black) and $\omega=0.06, \epsilon_{\|}=0.002$ (blue). (c) The scattering amplitude $f_{00}^{o}$ as a function of the scattering length $a_{p}$ for $\omega=0.002$ and $\epsilon_{\|}=0.0002$. The corresponding constant $V_{0}$ is varied in the region $-4.54<V_{0}<-4.47$. 
coefficient is plotted as a function of $\varepsilon$ for several values of $a_{p} / a_{\perp}$ for the two cases of being initially in the ground $n=0$ (a) and the first excited $n=1$ (b) transverse states for $\omega=0.002$. We observe that for any number of open channels, $T$ exhibits a minimum for some value of $a_{p} / a_{\perp}$. With increasing $a_{p} / a_{\perp}$ the transmission well becomes more shallow (except for the single-mode regime) and wider. In contrast to the bosonic case, there is no specific threshold behaviour. This is a consequence of the fact that the relative motion does not feel the interatomic interaction in the closed channels which are strongly screened by the centrifugal repulsion playing a dominant role for near-threshold collision energies or in other words: We encounter a weak coupling of the different scattering channels.
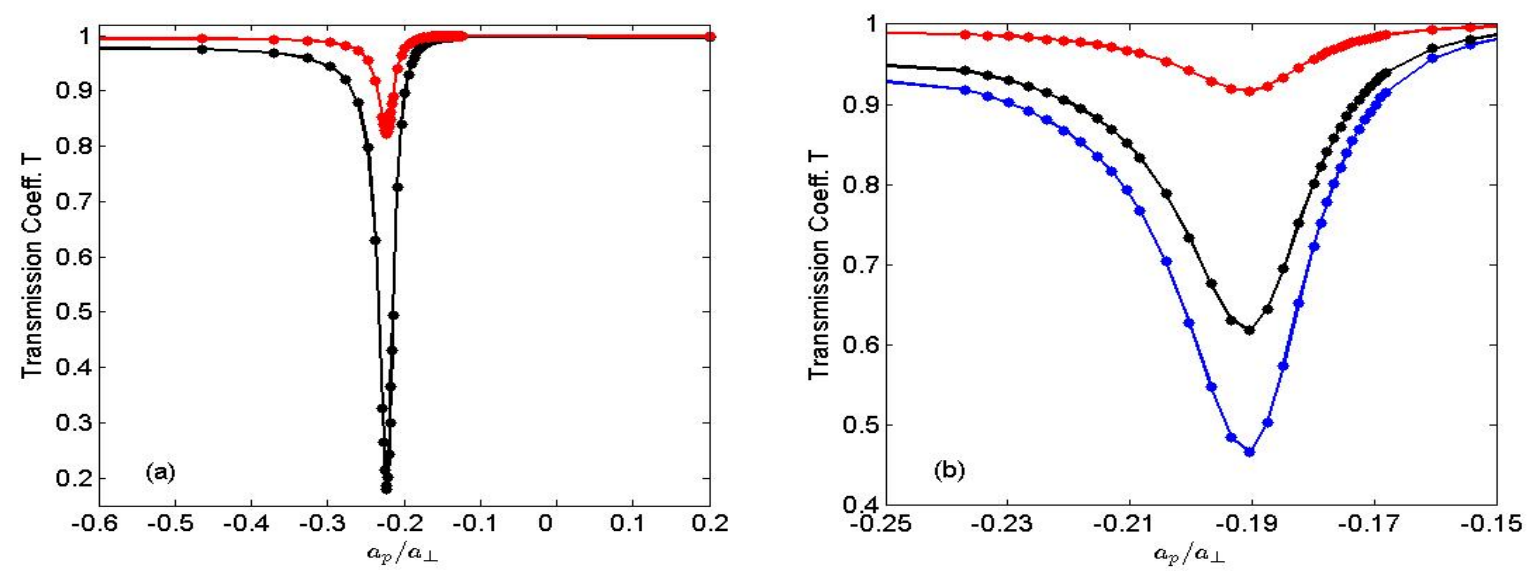

FIG. 11: Total transmission coefficient $T$ in a fermionic collision as a function of $a_{p}$ for $\omega=0.002$ for (a) two-open channels with $\varepsilon=1.05$ being initially in the ground state $n=0$ (black) and first excited state $n=1$ (red), and (b) three-open channel with $\varepsilon=2.05$ being initially in the ground state $n=0$ (blue), first excited state $n=1$ (black) and second excited state $n=2$ (red).

In Fig 13 we show the transition probabilities $W_{n n^{\prime}}$ as a function of $a_{p} / a_{\perp}$ for four open channels. We see that the probability of an elastic scattering process (i.e. to remain in the same transversal state) is much larger than that of an inelastic collision (i.e. the transition to a different transversal state). For an elastic collision the probability $W_{n n}$ shows a minimum and corresponding well which becomes wider and more shallow with increasing initial quantum number $n$ (i.e., with the population of a higher excited initial transversal state). For an inelastic collision $W_{n n^{\prime}}\left(n \neq n^{\prime}\right)$ exhibits a peak which becomes less pronounced as the quantum numbers $n$ or $n^{\prime}$ increase. 

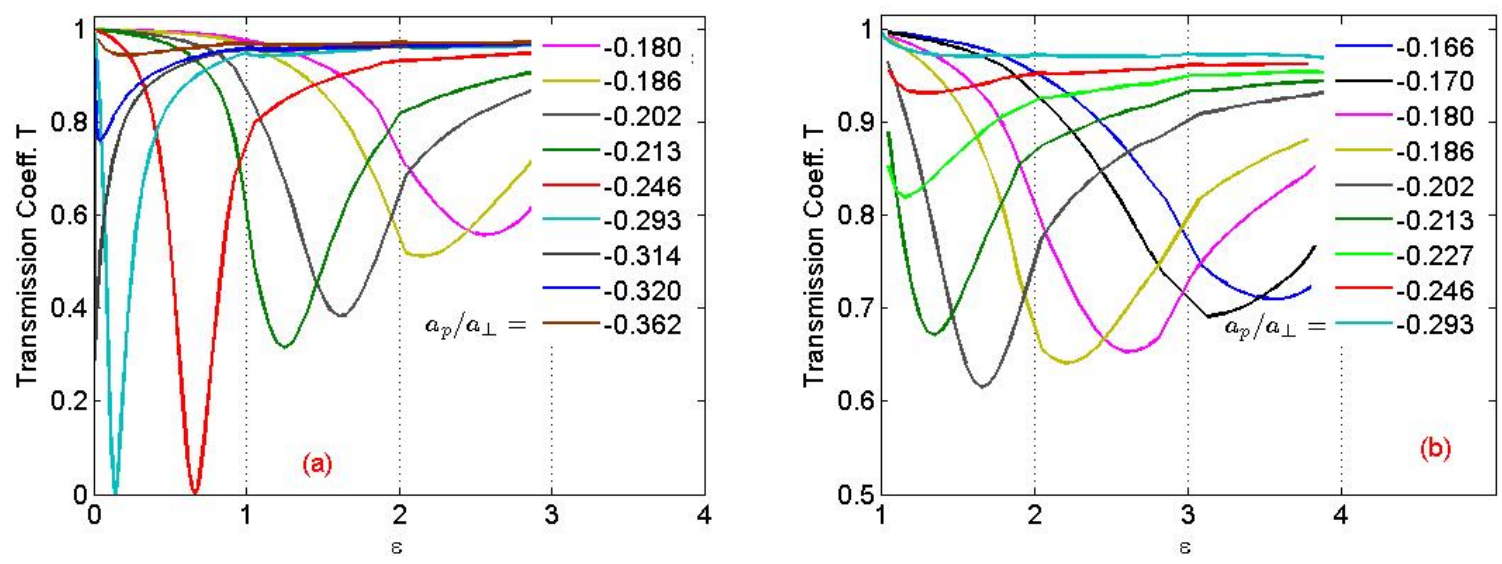

FIG. 12: Total transmission coefficient $T$ for a fermionic collision as a function of the dimensionless energy $\varepsilon$ for the two cases of the system being initially in the ground $n=0$ (a) and first excited $n=1$ (b) transverse states, for several ratios of $a_{p} / a_{\perp}$. We have used $\omega=0.002$.

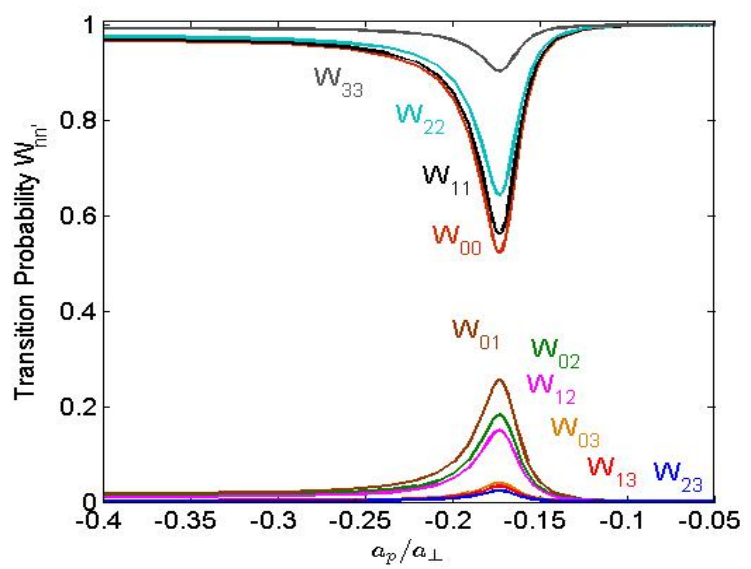

FIG. 13: Transition probabilities $W_{n n^{\prime}}$ in fermionic collisions as a function of $a_{p} / a_{\perp}$ for four open channels. $\omega=0.002$ and $\epsilon=0.0142$ are employed.

\section{Multi-channel scattering of distinguishable particles}

In this section we analyze the multi-channel scattering of two distinguishable particles in a harmonic trap with the same trap frequency $\omega_{1}=\omega_{2}=\omega$ allowing the separation of the c.m. motion. Such a case corresponds to the different atomic species confined by the same potential as it is the case e.g. for two different isotopes in the same optical dipole trap. The two-body scattering wave-function of the distinguishable atoms does not possess a well-defined symmetry with respect to the reflection $z \rightarrow-z$, i.e. both s- and p-state 
contributions $\left(f^{e}\right.$ and $\left.f^{o}\right)$ must be taken into account for the scattering amplitude. Fig. 14 shows our results for the transmission coefficient $T$ in the single-mode regime $(0<\varepsilon<1)$, which is plotted as a function of the tuning parameter $-V_{0}$ of the interparticle interaction. In general the scattering process can not be described by a single scattering length $a_{s}$ or $a_{p}$ for this case. In regions with a negligible p-wave contribution (see the regions in Fig, 1 with $\left.V_{p} \rightarrow 0\right), T$ exhibits a behavior similar to bosonic scattering. We observe the well-known s-wave CIRs which lead to zeros of the transmission $T$ at the positions $a_{s} / a_{\perp}=1 / C$ and tend to unity when $a_{s}$ goes to zero (together with $a_{p}$ ). In regions, where $a_{s}$ and $a_{p}$ are comparable we observe the effect reported in ref. [24]: remarkable peaks of the transmission $T=\left|1+f_{00}^{e}+f_{00}^{o}\right|^{2} \rightarrow 1$ i.e. almost complete transmission in spite of the strong interatomic interaction in free space. This is the so-called dual CIR: Quantum suppression of scattering in the presence of confinement due to destructive interference of odd and even scattering amplitudes. Equally minima of $T=\left|1+f_{00}^{e}+f_{00}^{o}\right|^{2} \rightarrow 0$ due to the interference of even and odd scattering amplitudes under the action of the transverse confinement can occur (see Fig, 14). Complete transmission corresponds to $f_{00}^{e}+f_{00}^{o}=-1-i$ while total reflection corresponds to $f_{00}^{e}+f_{00}^{o}=-1$. Fig 15 shows the corresponding amplitudes $f_{00}^{e}+f_{00}^{o}$ as a function of $-V_{0}$.

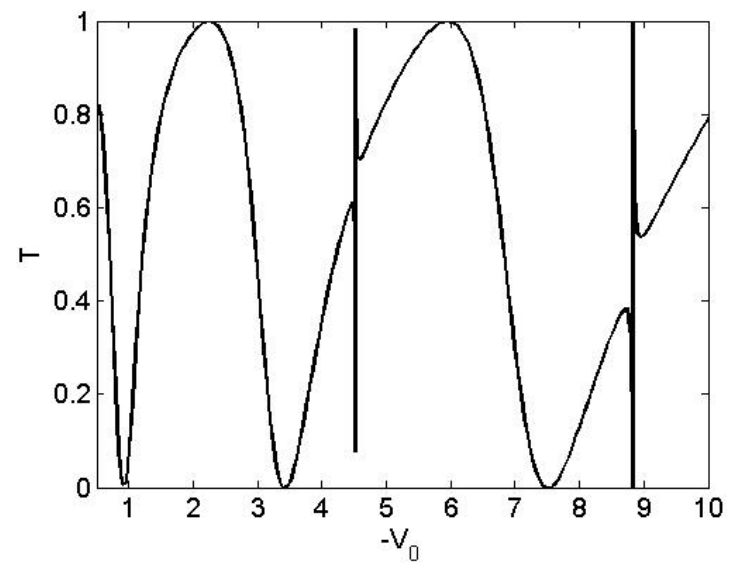

FIG. 14: Transmission $T$ as a function of the depth $-V_{0}$ of the potential (6) for two distinguishable atomic species for $\omega=0.002$ for $\epsilon_{\|}=0.0002$. Units according to eq.(7).

Fig 16 shows the total transmission coefficient versus $-V_{0}$ in the three-mode regime for $\omega=0.002$ and $\varepsilon=2.05$. Similar to the single-mode regime, when $a_{p}$ is negligible compared to $a_{s}, T$ behaves analogously to the case of a bosonic collision, and tends to unity (complete 


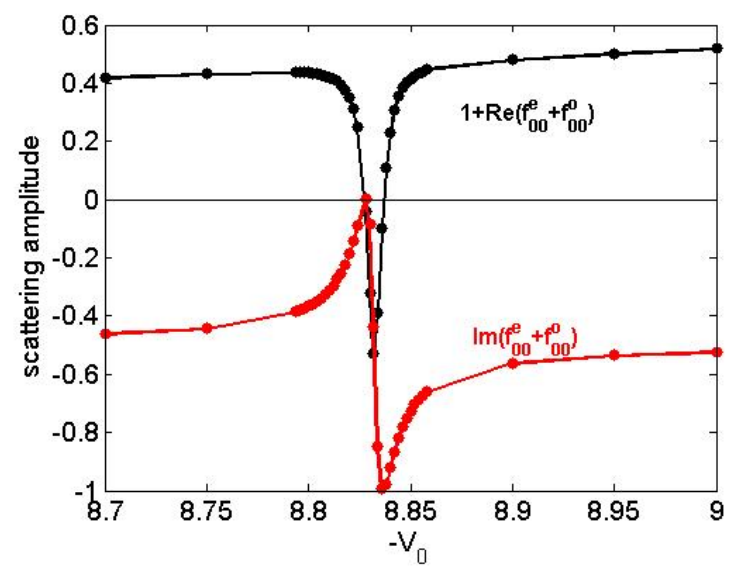

FIG. 15: Scattering amplitude as a function of the depth $-V_{0}$ of the potential (6) for two distinguishable atomic species for $\omega=0.002$ and $\epsilon_{\|}=0.0002$. Units according to eq. (7).

transmission) when $a_{s}$ tends to zero, while at the s-wave CIR position, it exhibits a minimum with a nonzero value. For a lower degree of transversal excitation of the initial state, we encounter a larger transmission coefficient. For the same reasons as in the single-mode regime, we observe in the regions of $V_{0}$ where the p-wave scattering length $a_{p}$ is comparable to $a_{s}$, sharp peaks of $T$. However in contrast to the single-mode regime we do not observe complete transmission i.e. $T \neq 1$ (see e.g. $T$ at $V_{0}=-8.85$ in Fig, 16).

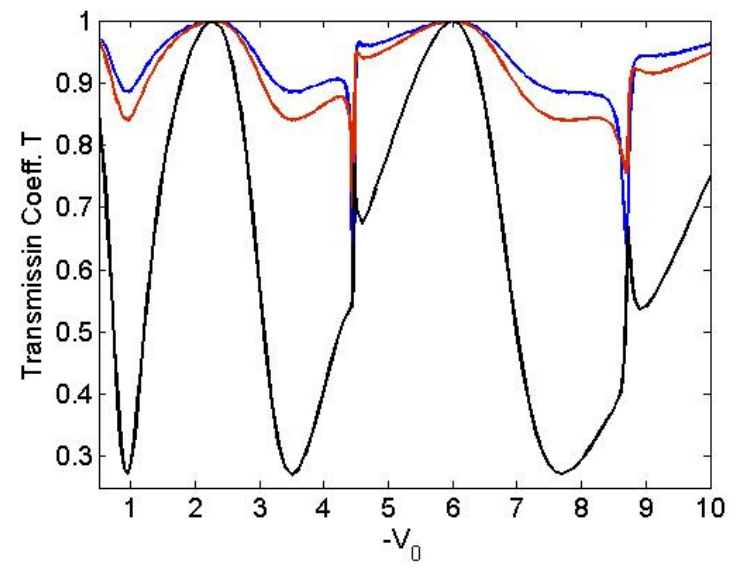

FIG. 16: Transmission coefficient $T$ as a function of the depth $-V_{0}$ of the potential (6) for two distinguishable atomic species for $\omega=0.002$ and $\varepsilon=2.05$ in the three-mode regime, being initially in transverse ground state (blue), first excited state (red) and second excited state (black). Units according to eq. (7). 
Fig 17 presents the transmission coefficients as a function of the dimensionless energy $\varepsilon$ for $\omega=0.002$. Fig 17 (a) shows the results for several ratios of $a_{s} / a_{\perp}$ if $\left|a_{p}\right|$ is small compare to $\left|a_{s}\right|$. Here the system is initially in the transversal ground state. The behaviour of the transmission coefficient is similiar to the case of two-boson scattering (see 6-a). Fig.17(b) shows the transmission for the scattering of two distinguishable atoms together with the results for bosonic- (blue) and fermionic- (black) collisions, for $-V_{0}=-4.505$ when both scattering length $a_{s}$ and $a_{p}$ are large. In the limit $\varepsilon \rightarrow 0$ the behaviour of the transmission for the distinguishable and the bosonic case are very similar while in the vicinity of the energy of the p-wave shape-resonance $\varepsilon \sim 0.7$, apart from a small shift to larger energies, we can find a complete coincidence of the transmission behaviour for the case of distinguishable and fermionic scattering. At the threshold energy $\varepsilon=1$ the latter two transmission curves cross. Fig 17 (c) shows the transmission coefficient versus $\varepsilon$ for $-V_{0}=-4.505$ initially occupying the ground state $n=0$ (blue), the first excited state $n=1$ (black), the second excited state $n=2$ (red) and the third excited state $n=3$ (green). In the limit $\varepsilon_{\|} \rightarrow 0, T$ drops rapidly to zero. Apart from the single mode regime, it is for $\varepsilon_{\|}>>0$ approximately constant with $T \approx 1$.

In fig. 18 we present the transition probabilities $W_{n n^{\prime}}$ as a function of $-V_{0}$ for four open channels. For the regions of $V_{0}$ where $a_{p}$ is negligible compared to $a_{s}$, a very good agreement of the transmission behaviour for distinguishable and bosonic atoms is observed. In regions where $a_{s}$ and $a_{p}$ are comparable, similar to the fermionic case, we observe narrow and deep wells (for elastic collision) and narrow as well as strongly pronounced peaks (for inelastic collision).

\section{SUMMARY AND CONCLUSIONS}

We have analyzed atomic multi-channel scattering in a 2D harmonic confinement. Identical bosonic and fermionic scattering as well as scattering of distinguishable atoms in traps with the same frequency for the different species have been explored. Equal frequencies allows us to separate the c.m. and relative motion.

Firstly we reproduced the well-known s-wave CIR for bosonic collision in the single-mode regime [13, 14, 24, 25, 26, 29]. Next bosonic collisions in the multi-channel regime including elastic and inelastic processes i.e. transverse excitations and deexcitations have been in- 

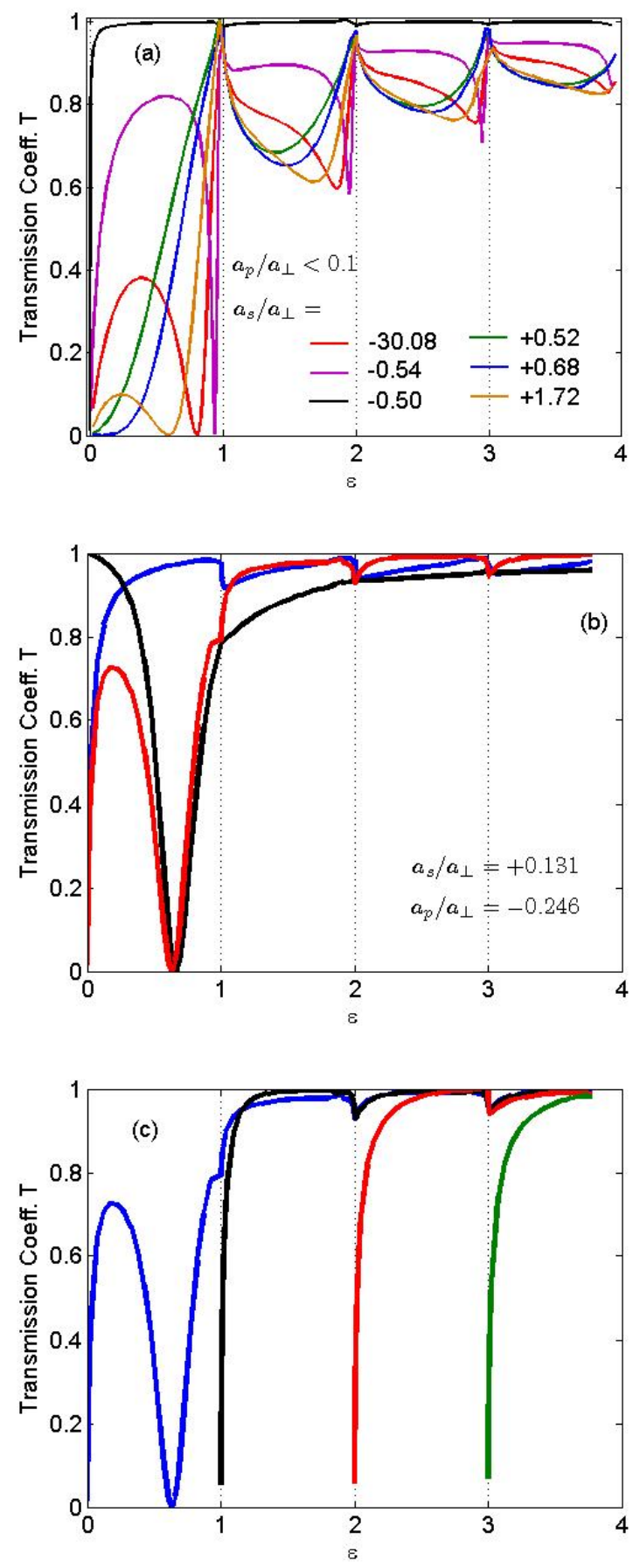

FIG. 17: Transmission coefficient $T$ as a function of the dimensionless energy $\varepsilon$ for scattering of two distinguishable particles in a harmonic confinement $\omega=0.002$ (a) for several ratios $a_{s} / a_{\perp}$ in the case where $a_{p}$ is negligible compared to $a_{s}$, (b) for $-V_{0}=-4.505$ when both scattering lengths $a_{s}$ and $a_{p}$ are comparable (here the transmission coefficients for bosonic- (blue) and fermionic(black) collision are also provided) and (c) for $-V_{0}=-4.505$ when the system is initially in the ground transversal state (blue), in the first excited state (black), in the second excited state (red) and in the third excited state (green). 


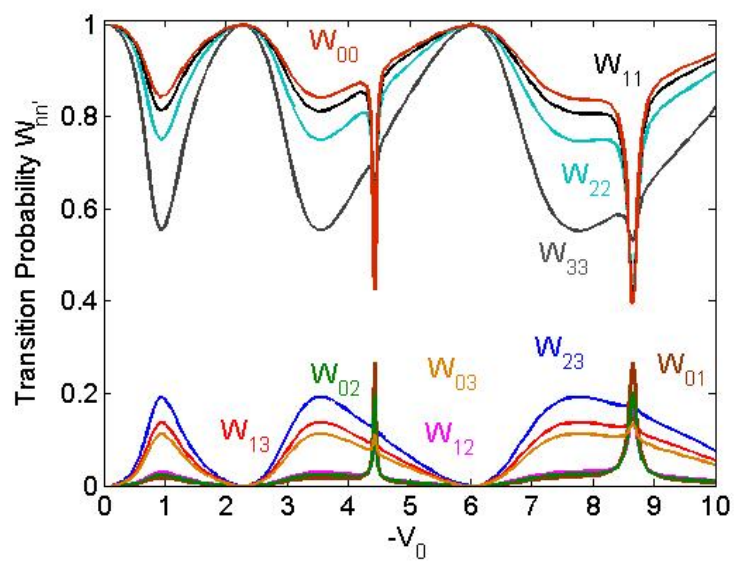

FIG. 18: Transition probabilities $W_{n n^{\prime}}$ for quasi-1D scattering of distinguishable particles in a harmonic trap $\omega=0.002$, as a function of $-V_{0}$ for four open channels and $\varepsilon=2.05$. $V_{0}$ is given in units of eq.(7).

vestigated. Transmission coefficients as well as transition probabilities for energies covering up to four open channels are reported on. It is shown that the transmission coefficient as a function of the scattering length exhibits a minimum at the CIR position. Except for $0<a_{s} / a_{\perp}<1 / C$ in the first channel, the transmission curves show, with varying energy, a minimum and accompanying well. For a single open channel the value of the minimum of the transmission is zero. Increasing the degree of transverse excitation leads to an increase of the transmission minima. A distinct threshold behaviour is observed at every $\varepsilon=n$.

For $a_{s} / a_{\perp}<0$ the accompanying transmission well is more pronounced compared to the case $a_{s} / a_{\perp}>0$ and it is also deeper in the case of several open channels. The position of the minimum is closer to the upper threshold again when compared to the case $a_{s} / a_{\perp}>$ 0 . With increasing ratio $a_{s} / a_{\perp}$ for either $a_{s} / a_{\perp}>0$ or $a_{s} / a_{\perp}<0$, the corresponding transmission well becomes also more pronounced i.e. we encounter a deeper and narrower well for more open channels and the energetical position of the minimum moves to the neighboring upper threshold. Our results for the transition probabilities $W_{n n^{\prime}}$ show that the probability of remaining in the same state (elastic collision) is larger than transitions into a different transversal state (inelastic collision). In the case of elastic collisions (inelastic collisions) it has to go to unity (zero) as $a_{s}$ tends to zero. The inelastic transition probability $W_{n n^{\prime}}$ increases as the numbers $n$ or $n^{\prime}$ increase while the elastic transition probability $W_{n n^{\prime}}$ decreases. 
Our next focus has been the multi-channel scattering of fermions addressing up to four open channels in the waveguide. In the regime of a single open channel we reproduced the well-known p-wave mapped CIR in the zero-energy limit [18, 26]. We have analyzed the pwave CIR dependence on the collision energy $\epsilon$ and the trapping frequency $\omega$. Consequently the multi-mode regime has been explored by determining the behaviour of the transmission as well as the transition probabilities. The dependence of the transmission on the p-wave scattering length exhibits a minimum with zero transmission and an accompanying well for the single-mode regime. Increasing the energy, this well becomes more shallow (except for the single-mode regime), wider and its position is shifted to the region of higher energies. In the multi-mode regime the transmission well depends also on the degree of the initial transverse excitation. Increasing the degree of initial transverse excitation the transmission well becomes shallower and wider and the transmission is overall increased. For a fixed number of open channels the transmission exhibits as a function of the energy a minimum for some value of the p-wave scattering length. Increasing $a_{p} / a_{\perp}$ leads to a shift of the transmission minimum to higher energies and an increasingly shallower well. This holds except for the single mode regime in which the minimal value is zero.

In contrast to the bosonic case, we do not observe a distinct threshold behavior. The transition probabilities $W_{n n^{\prime}}$ for fermionic collisions show that elastic collisions are more probable than inelastic ones. The probability $W_{n n}$ for an elastic process shows a well which becomes wider and shallower as the initial population of the excited states $n$ increases, while for an inelastic collision it exhibits a peak which becomes smaller as the channel numbers $n$ or $n^{\prime}$ increase. By varying the potential parameter $V_{0}$ in the viccinity of the value $V_{0}=-4.54$, we found a set of shape-resonances.

Finally we analyzed the multi-channel-1D scattering of distinguishable atoms in the trap. Here both s- and p-wave contributions have been taken into account. We have studied the transmission coefficient in the single-mode regime and reproduced the CIR [13, 14, 18, 29] as well as the dual-CIR [24, 25, 26] corresponding to total reflection and transmission, respectively. In the multi-mode regime the transmission versus $-V_{0}$ shows except for the regions where $a_{p}$ is comparable with $a_{s}$ a behaviour analogous to the situation of bosonic scattering. We encounter $T \rightarrow 0$ for $a_{s} \rightarrow 0$. At the position of the CIR, $T$ exhibits a minimum with a nonzero value. The lower is the transverse excitation of the initial state, the larger is the transmission coefficient. In regions of $V_{0}$ where the p-wave scattering length 
$a_{p}$ is comparable to $a_{s}, T$ exhibits sharp peaks or dips near the dual-CIRs due to comparable contributions of both s- and p-waves. In contrast to the single-mode regime there is no a complete transmission at the dual CIR points. Our results for the transmission coefficient versus energy show that in the limit of zero longitudinal relative energy the distinguishable atoms behave like bosons, while in the vicinity of the position of a shape-resonance, they behave like fermions. For larger energies $\varepsilon>>1 T$ is close to unity. Finally we have analyzed the transition probability as a function of $-V_{0}$. For values of $V_{0}$ where $a_{p}$ is negligible compared to $a_{s}$ we find an excellent agreement with the results for bosonic collisions. In regions where these two scattering parameters are comparable, we observe, similar to the fermionic case, sharp downward- (for elastic collision) and upward- (for inelastic collision) peaks but with different values. We conclude with the general statement that our multichannel scattering results in waveguides are of immediate relevance to cold or ultracold atomic collisions in atomic waveguides or impurity scattering in quantum wires.

\section{ACKNOWLEDGMENTS}

S.S. acknowledges a scholarship by the Ministry of Science, Research and Technology of Iran. V.S.M. acknowledges financial support by the Landesstiftung Baden-Württemberg in the framework of a guest program. Financial support by the Heisenberg-Landau Program is also acknowledged. P.S. thanks the Deutsche Forschungsgemeinschaft for financial support.

\section{APPENDIX A. BOUNDARY CONDITIONS}

In the limit $r$ tends to zero, $\mathbf{u}$ goes to zero. This boundary condition can be satisfied easily by putting $\mathbf{u}(r=0)=\mathbf{0}$ and $\mathbf{u}_{-j}=\mathbf{u}_{j}$ in the vicinity of $r=0$. The latter is needed for approximating the derivatives $\frac{d^{2}}{d x^{2}} u(r(x))$ and $\frac{d}{d x} u(r(x))$ near the point $r=0$.

The boundary condition for $u$ approximating the asymptotic form (13) at large $r$ can be written in the form

$$
\mathbf{u}_{j}+\alpha_{j}^{(1)} \mathbf{u}_{j-1}+\alpha_{j}^{(2)} \mathbf{u}_{j-2}+\alpha_{j}^{(3)} \mathbf{u}_{j-3}+\alpha_{j}^{(4)} \mathbf{u}_{j-4}=\mathbf{g}_{j} \quad j=N-2, N-1, N
$$

where $\alpha_{j}$ s are diagonal $N_{\theta} \times N_{\theta}$ matrices and $\mathbf{g}_{j}$ is a $N_{\theta}$-dimensional vector. The above boundary-conditions are constructed by eliminating the unknown amplitudes $f_{n n^{\prime}}$ from the 
asymptotic equations (13) written for a few $r_{j}$ s neighbouring to the point $r_{N}=R$. This gives the values for the coefficients $\alpha_{j}$ s and $\mathbf{g}_{j}$. In general for up to four open channels we have

$$
\left[\alpha_{j}^{(l)}\right]_{m m^{\prime}}=(-1)^{i}\left(T_{j}^{l-1, m}+T_{j}^{l, m}\right) \frac{r_{j}}{r_{j-l}} \frac{\phi_{n_{e}}\left(\rho_{j}^{m}\right)}{\phi_{n_{e}}\left(\rho_{j-l}^{m}\right)} e^{i k_{n_{e}}\left(\left|z_{j}^{m}\right|-\left|z_{j-l}^{m}\right|\right)} \delta_{m m^{\prime}}, \quad l=1,2,3,4
$$

and

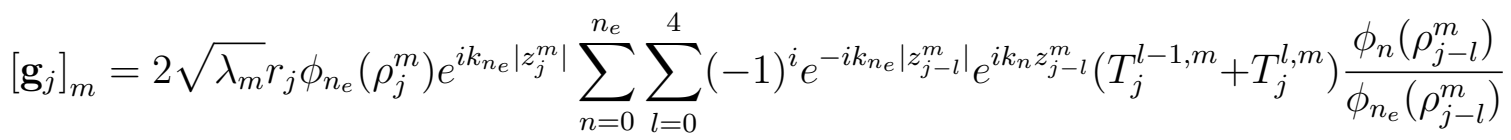

For the case of bosonic (fermionic) collisions we must consider just the even (odd) part of the equation 30 , i.e. we just need to replace the term $e^{i k_{n} z_{j-l}^{m}}$ by $\cos k_{n} z_{j-l}^{m}\left(i \sin k_{n} z_{j-l}^{m}\right)$. Here $\phi_{n}(\rho)=\phi_{n, 0}(\rho, \varphi)$ is the eigenfunction of the transverse trapping Hamiltonian (see Eq.(9p), $\rho_{j}^{m}=r_{j} \sin \theta_{m}, z_{j}^{m}=r_{j} \cos \theta_{m}, n$ is the channel number of the initial state, and $n_{e}$

is the number of transversely excited open channels. The complex numbers $T_{j}^{i, m} \mathrm{~S}$ are given through

$$
T_{j}^{l, m}=\delta_{l, 0}+\delta_{l, 1}\left(a_{j}^{m}+b_{j}^{m}+c_{j}^{m}\right)+\delta_{l, 2}\left(a_{j}^{m} b_{j-1}^{m}+a_{j}^{m} c_{j-1}^{m}+b_{j}^{m} c_{j-1}^{m}\right)+\delta_{l, 3} a_{j}^{m} b_{j-1}^{m} c_{j-2}^{m}
$$

If $n_{e}<1$ (a single-mode regime) $a_{j}^{m}=0$, otherwise

$$
\begin{aligned}
a_{j}^{m}= & \left\{\Xi_{0, j}^{m}-\left(1+b_{j}^{m}+c_{j}^{m}\right) \Xi_{0, j-1}^{m}+\left(b_{j}^{m}+c_{j}^{m}+b_{j}^{m} c_{j-1}^{m}\right) \Xi_{0, j-2}^{m}-b_{j}^{m} c_{j-1}^{m} \Xi_{0, j-3}^{m}\right\} \times \\
& \left\{\Xi_{0, j-1}^{m}-\left(1+b_{j}^{m}+c_{j}^{m}\right) \Xi_{0, j-2}^{m}+\left(b_{j}^{m}+c_{j}^{m}+b_{j}^{m} c_{j-1}^{m}\right) \Xi_{0, j-3}^{m}-b_{j}^{m} c_{j-1}^{m} \Xi_{0, j-4}^{m}\right\}^{-1}
\end{aligned}
$$

if $n_{e}<2, b_{j}^{m}=0$, otherwise

$$
b_{j}^{m}=\left\{\Xi_{1, j}^{m}-\left(1+c_{j}^{m}\right) \Xi_{1, j-1}^{m}+c_{j}^{m} \Xi_{1, j-2}^{m}\right\} \times\left\{\Xi_{1, j-1}^{m}-\left(1+c_{j}^{m}\right) \Xi_{1, j-2}^{m}+c_{j}^{m} \Xi_{1, j-3}^{m}\right\}^{-1}
$$

if $n_{e}<3, c_{j}^{m}=0$, otherwise

$$
c_{j}^{m}=\left\{\Xi_{2, j}^{m}-\Xi_{2, j-1}^{m}\right\} \times\left\{\Xi_{2, j-1}^{m}-\Xi_{2, j-2}^{m}\right\}^{-1}
$$

there $\Xi_{n, j}^{m}=e^{i\left(k_{n}-k_{n_{e}}\right)\left|z_{j}^{m}\right|} \frac{\phi_{n}\left(\rho_{j}^{m}\right)}{\phi_{n_{e}}\left(\rho_{j}^{m}\right)} . k_{n}$ and $k_{n_{e}}$ are given by eq. 12 .

\section{APPENDIX B. FAST IMPLICIT MATRIX ALGORITHM}

Following the idea of the LU-decomposition [33] and the sweep method [34] (or the Thomas algorithm [35]), we search the solution of the system of $N$ vector equations with any coefficient being a $N_{\theta} \times N_{\theta}$ matrix (27) in the form

$$
\mathbf{u}_{j}=C_{j}^{(1)} \mathbf{u}_{j+1}+C_{j}^{(2)} \mathbf{u}_{j+2}+C_{j}^{(3)} \mathbf{u}_{j+3}, \quad j=1, \ldots, N-3
$$


Here we define $\mathbf{u}\left(r\left(x_{j}\right)\right)$ as $\mathbf{u}_{j}$ for simplicity. The $C_{j}$ s are unknown $N_{\theta} \times N_{\theta}$ matrices. To find the solution, first we should calculate the unknown $C_{j}$ matrices. The plan is the following: Due to eq. 35 we have

$$
\mathbf{u}_{j-p}=C_{j-p}^{(1)} \mathbf{u}_{j-p+1}+C_{j-p}^{(2)} \mathbf{u}_{j-p+2}+C_{j-p}^{(3)} \mathbf{u}_{j-p+3}, \quad p=1,2,3,
$$

then one obtains

$$
\begin{gathered}
\mathbf{u}_{j-1}=C_{j-1}^{(1)} \mathbf{u}_{j}+C_{j-1}^{(2)} \mathbf{u}_{j+1}+C_{j-1}^{(3)} \mathbf{u}_{j+2} \\
\mathbf{u}_{j-2}=\left[C_{j-2}^{(1)} C_{j-1}^{(1)}+C_{j-2}^{(2)}\right] \mathbf{u}_{j}+\left[C_{j-2}^{(1)} C_{j-1}^{(2)}+C_{j-2}^{(3)}\right] \mathbf{u}_{j+1}+C_{j-2}^{(1)} C_{j-1}^{(3)} \mathbf{u}_{j+2}
\end{gathered}
$$

and

$$
\begin{array}{r}
\mathbf{u}_{j-3}=\left[C_{j-3}^{(1)} C_{j-2}^{(1)} C_{j-1}^{(1)}+C_{j-3}^{(1)} C_{j-2}^{(2)}+C_{j-2}^{(2)} C_{j-1}^{(1)}+C_{j-2}^{(3)}\right] \mathbf{u}_{j} \\
+\left[C_{j-3}^{(1)} C_{j-2}^{(1)} C_{j-1}^{(2)}+C_{j-3}^{(1)} C_{j-2}^{(3)}+C_{j-2}^{(2)} C_{j-1}^{(2)}\right] \mathbf{u}_{j+1} \\
+\left[C_{j-3}^{(1)} C_{j-2}^{(1)} C_{j-1}^{(3)}+C_{j-2}^{(2)} C_{j-1}^{(3)}\right] \mathbf{u}_{j+2}
\end{array}
$$

By substituting $u_{j}$ defined by Eqs. (37.39) into Eq. 27) one can calculate $\mathbf{u}_{j}$ in terms of $\mathbf{u}_{j+1}, \mathbf{u}_{j+2}$ and $\mathbf{u}_{j+3}$. Then, by comparing with Eq. 35. we find a recurrence formula for calculating the unknown matrices $C_{j}$ :

$$
\begin{aligned}
-D C_{j}^{(1)}= & \mathbb{A}_{j-3}^{j}\left[C_{j-3}^{(1)} C_{j-2}^{(1)} C_{j-1}^{(2)}+C_{j-3}^{(1)} C_{j-2}^{(3)}+C_{j-3}^{(2)} C_{j-1}^{(2)}\right] \\
+ & \mathbb{A}_{j-2}^{j}\left[C_{j-2}^{(1)} C_{j-1}^{(2)}+C_{j-2}^{(3)}\right]+\mathbb{A}_{j-1}^{j} C_{j-1}^{(2)}+\mathbb{A}_{j+1}^{j} \\
-D C_{j}^{(2)}= & \mathbb{A}_{j-3}^{j}\left[C_{j-3}^{(1)} C_{j-2}^{(1)} C_{j-1}^{(3)}+C_{j-3}^{(2)} C_{j-1}^{(3)}\right] \\
& +\mathbb{A}_{j-2}^{j} C_{j-2}^{(1)} C_{j-1}^{(3)}+\mathbb{A}_{j-1}^{j} C_{j-1}^{(3)}+\mathbb{A}_{j+2}^{j}
\end{aligned}
$$

and

$$
-D C_{j}^{(3)}=\mathbb{A}_{j+3}^{j}
$$

Here

$$
\begin{aligned}
D & =\mathbb{A}_{j-3}^{j}\left[C_{j-3}^{(1)} C_{j-2}^{(1)} C_{j-1}^{(1)}+C_{j-3}^{(1)} C_{j-2}^{(2)}+C_{j-3}^{(2)} C_{j-1}^{(1)}+C_{j-3}^{(3)}\right] \\
& +\mathbb{A}_{j-2}^{j}\left[C_{j-2}^{(1)} C_{j-1}^{(1)}+C_{j-2}^{(2)}\right]+\mathbb{A}_{j-1}^{j} C_{j-1}^{(1)}+\mathbb{A}_{j}^{j}+2\left(\epsilon I-V_{j}\right)
\end{aligned}
$$

By using the left-side boundary conditions and Eq. 35) one can calculate the $C_{j}$ matrices for $j=1,2$ and 3 . Then by using Eqs. 43,40 we calculate all the matrices $C_{j}$. Subsequently by 
using the right-side boundary conditions 28 and recurrence formula (35) we first calculate $\mathbf{u}_{j}$ for $j=N-2, N-1$ and $N$ and then $\mathbf{u}_{j}$ for $j=1, \ldots, N-3$.

[1] R. Grimm, M. Weidemüller, and Y.B. Ovchinnikov, Adv. At. Mol. Opt. Phys. 42, 95 (2000).

[2] R. Folman et al.,Adv. At. Mol. Opt. Phys. 42, 95 (2000).

[3] J. Reichel, Appl. Phys. B: Laser Opt. 74, 469 (2002).

[4] J. Fortagh et al., Rev. Mod. Phys. 79, 235 (2007).

[5] E.L. Bolda, E. Tiesinga and P.S. Julienne, Phys.Rev.A 66, 013403 (2002).

[6] R. Stock, I.H. Deutsch and E.L. Bolda, Phys.Rev.Lett.91, 183201 (2003).

[7] V.A. Yurovsky, Phys.Rev.A71, 012709 (2005).

[8] R. Stock and I.H. Deutsch, Phys.Rev.A 73, 32701 (2006).

[9] V.A. Yurovsky and Y.H. Band, Phys.Rev.A 75, 012717 (2007).

[10] P. Naidon et al, New J. Phys. 9, 19 (2007).

[11] S.G. Bhongale, S.J.J.M.F. Kokkelmans and I.H. Deutsch, arXiv:0712.2070v1, physics:atomph.

[12] V.A. Yurovsky, M. Ol'shanii and D.S. Weiss, Adv.At.Mol.Opt.Phys. 55, 61 (2007).

[13] M. Olshanii, Phys. Rev. Lett. 81, 938 (1998).

[14] T. Bergeman, M.G. Moore, and M. Olshanii, Phys. Rev. Lett. 91, 163201 (2003).

[15] C. Mora, R. Egger, A.O. Gogolin, and A. Komnik, Phys. Rev. Lett. 93, 170403 (2004).

[16] C. Mora, R. Egger, and A.O. Gogolin, Phys. Rev. A71 052705 (2005).

[17] C. Mora, A. Komnik, R. Egger, and A.O. Gogolin, 2005 Preprint cond-mat/0501641.

[18] B.E. Granger and D. Blume, Phys.Rev. Lett. 92, 133202 (2004).

[19] T. Kinoshita, T. Wenger and D.S. Weiss, Science 305, 1125 (2004).

[20] B. Paredes et al, Nature 429, 277 (2004).

[21] K. Günter et al, Phys. Rev. Lett. 95, 230401 (2005).

[22] J.I. Kim, J. Schmiedmayer, and P. Schmelcher, Phys. Rev. A72, 042711 (2005).

[23] V. Peano, M. Thorwart, C. Mora, and R. Egger, New J. Phys. 7, 1 (2005).

[24] J.I. Kim, V.S. Melezhik, and P. Schmelcher, Phys. Rev. Lett. 97, 193203 (2006).

[25] J.I. Kim, V.S. Melezhik, and P. Schmelcher, Rep. Progr. Theor. Phys. Supp. 166, 159 (2007).

[26] V.S. Melezhik, J.I. Kim, and P. Schmelcher, Phys. Rev. A76, 053611 (2007). 
[27] A. Lupu-Sax, Quantum Scattering Theory and Applications, PhD Thesis, Harvard University (1998).

[28] M.G.E. da Luz, A.S. Lupu-Sax and E.J. Heller, Phys.Rev.E 56, 2496 (1997)

[29] M.G. Moore, T. Bergeman and M. Olshanii, J. Phys. IV 116, 69 (2004), Lecture courses Les Houches School on 'Quantum Gases in Low Dimensions' (2003).

[30] V.S. Melezhik, J. Comput. Phys. 92, 67 (1991).

[31] V.S. Melezhik and Chi-Yu Hu, Phys. Rev. Lett. 90, 083202 (2003).

[32] V.S. Melezhik, Phys. Lett. A230, 203 (1997).

[33] W.H. Press, S.A. Teukolsky, W.T. Vetterling, and B.P. Flannery, Numerical Recipes, Cambridge University Press, 1992.

[34] I.M. Gelfand and S.V. Fomin, Calculus of Variations, Dover Publications, 2000.

[35] G.N. Bruse et al., Petrol. Trans. AIME 198, 79 (1953). 